\title{
Complete surfaces with positive extrinsic curvature in product spaces
}

\author{
José M. Espinar, José A. Gálvez and Harold Rosenberg*
}

\begin{abstract}
We prove that every complete connected immersed surface with positive extrinsic curvature $K$ in $\mathbb{H}^{2} \times \mathbb{R}$ must be properly embedded, homeomorphic to a sphere or a plane and, in the latter case, study the behavior of the end. Then, we focus our attention on surfaces with positive constant extrinsic curvature ( $K$-surfaces). We establish that the only complete $K$-surfaces in $\mathbb{S}^{2} \times \mathbb{R}$ and $\mathbb{H}^{2} \times \mathbb{R}$ are rotational spheres. Here are the key steps to achieve this. First height estimates for compact $K$-surfaces in a general ambient space $\mathbb{M}^{2} \times \mathbb{R}$ with boundary in a slice are obtained. Then distance estimates for compact $K$-surfaces (and $H$-surfaces) in $\mathbb{H}^{2} \times \mathbb{R}$ with boundary on a vertical plane are obtained. Finally we construct a quadratic form with isolated zeroes of negative index.
\end{abstract}

Mathematics Subject Classification (2000). 53C42, 53C40.

Keywords. Homogeneous product spaces, positive extrinsic curvature, Hadamard-Stoker type theorem, height estimates, classification of $K$-surfaces.

\section{Introduction}

In 1936, J. Stoker [S] generalized the result of J. Hadamard [H] that a compact strictly locally convex surface in Euclidean 3 -space $\mathbb{R}^{3}$ is homeomorphic to the sphere. J. Stoker showed that a complete strictly locally convex immersed surface in $\mathbb{R}^{3}$ must be embedded and homeomorphic to the sphere or plane. In the latter case, the surface is a graph over a planar domain. Today, this result is known as the Hadamard-Stoker Theorem. Strict convexity of the surface is equivalent in $\mathbb{R}^{3}$ to positive Gaussian curvature. Note that in $\mathbb{R}^{3}$, the Gauss equation for a surface says that the Gauss curvature, i.e., the intrinsic curvature $K(\mathrm{I})$, and the Gauss-Kronecker curvature, i.e., the extrinsic curvature $K$, are equal. In space forms, the situation is close since both curvatures are related by a constant.

M. Do Carmo and F. Warner [CW] extended Hadamard's theorem to hyperbolic 3 -space $\mathbb{H}^{3}$, assuming the surface is compact and has positive extrinsic curvature.

\footnotetext{
*J. M. Espinar and J.A. Gálvez were partially supported by Ministerio de Educación y Ciencia Grant No. MTM2007-65249 and Junta de Andalucía projects FQM325 and P06-FQM-01642.
} 
The complete case in $\mathbb{H}^{3}$ was treated by R. J. Currier in $[C]$ and it is interesting to remark the difference with the euclidean case. Currier's theorem says that a complete immersed surface in $\mathbb{H}^{3}$ whose principal curvatures are greater than or equal to one is embedded and homeomorphic to the sphere or plane. And we cannot expect a better result; it is easy to construct examples of complete embedded flat surfaces, i.e., $K=1$ in $\mathbb{H}^{3}$, homeomorphic to a cylinder.

Recently, the study of surfaces in product spaces $\mathbb{M}^{2} \times \mathbb{R}$, where $\mathbb{M}^{2}$ is a Riemannian surface and $\mathbb{R}$ the real line, has undergone considerable development. U. Abresch and H. Rosenberg [AR1] defined a holomorphic quadratic differential on constant mean curvature surfaces in the homogeneous 3-manifolds. This enabled them to generalize Hopf's theorem to these spaces: immersed constant mean curvature spheres are rotational and embedded. Aledo, Espinar and Gálvez associated a holomorphic quadratic differential to constant Gaussian curvature surfaces in $\mathbb{S}^{2} \times \mathbb{R}$ and $\mathbb{H}^{2} \times \mathbb{R}$, [AEG1]. This enabled them to prove the same Hopf type theorem for immersed constant Gaussian spheres in these product spaces. Also, they classified the complete surfaces with constant Gauss curvature, and established Liebmann and Hilbert type theorems for these surfaces. More precisely:

Liebmann type theorem. There exists a unique complete surface of constant Gaussian curvature $K(\mathrm{I})>1$ in $\mathbb{S}^{2} \times \mathbb{R}$ (up to isometry), and a unique complete surface of constant Gaussian curvature $K(\mathrm{I})>0$ in $\mathbb{H}^{2} \times \mathbb{R}$. In addition, these surfaces are rotationally symmetric embedded spheres.

Hilbert type theorem. There is no complete immersion of constant Gaussian curvature $K(\mathrm{I})<-1$ into $\mathbb{H}^{2} \times \mathbb{R}$ or $\mathbb{S}^{2} \times \mathbb{R}$.

Also, there exist complete immersions of every constant curvature $K(\mathrm{I}) \geq-1$ into $\mathbb{H}^{2} \times \mathbb{R}$. In [AEG3] the authors prove that there are no complete immersions with constant Gaussian curvature $0<K(\mathrm{I})<1$ in $\mathbb{S}^{2} \times \mathbb{R}$. The existence of complete immersions with constant Gaussian curvature $-1 \leq K(\mathrm{I})<0$ in $\mathbb{S}^{2} \times \mathbb{R}$ remains open.

In contrast, the case of extrinsic curvature has been rarely considered in these spaces (see [CR]). We note that the classification of surfaces of constant Gaussian curvature does not help us since the intrinsic and extrinsic curvature differ by the sectional curvature function in a product space.

We center our attention on complete surfaces with positive (non constant and constant) extrinsic curvature in $\mathbb{H}^{2} \times \mathbb{R}$, nonetheless some of our results for constant extrinsic curvature also work in a more general setting, as we will point out.

One of the main results of this paper is that only embedded rotational spheres can occur when $K$ is a positive constant, see Theorem 7.3.

We organize the paper as follows. In Section 2 we introduce the notation and definitions we need. In Section 3 we establish the following Hadamard-Stoker type 
theorem in $\mathbb{H}^{2} \times \mathbb{R}$ (the notion of simple end will be given later).

Theorem 3.1. Let $S$ be a complete connected immersed surface with $K>0$ in $\mathbb{H}^{2} \times \mathbb{R}$. Then $S$ must be properly embedded and bounds a strictly convex domain in $\mathbb{H}^{2} \times \mathbb{R}$. Moreover, $S$ is homeomorphic to $\mathbb{S}^{2}$ or $\mathbb{R}^{2}$. In the latter case, $S$ is a graph over a convex domain of $\mathbb{H}^{2} \times\{0\}$ or $S$ has a simple end.

This result suggests that surfaces with positive extrinsic curvature in $\mathbb{H}^{2} \times \mathbb{R}$ behave like surfaces with $K>0$ in $\mathbb{R}^{3}$, rather than surfaces with $K>0$ in $\mathbb{H}^{3}$. This is because there are many totally geodesic foliations of $\mathbb{H}^{2} \times \mathbb{R}$ by vertical planes which are isometric to $\mathbb{R}^{2}$.

In Section 4 we construct complete embedded surfaces with positive extrinsic curvature with a simple end in $\mathbb{H}^{2} \times \mathbb{R}$. In Section 5 we classify the complete revolution surfaces of positive constant extrinsic curvature in $\mathbb{H}^{2} \times \mathbb{R}$ which are topological spheres. Hereafter we will refer to surfaces with positive constant extrinsic curvature as $K$-surfaces.

In Section 6 we establish vertical height estimates for $K$-surfaces in $\mathbb{M}^{2} \times \mathbb{R}, \mathbb{M}^{2}$ a Riemannian surface. More precisely,

Theorem 6.1. Let $\psi: S \rightarrow \mathbb{M}^{2} \times \mathbb{R}$ be a compact graph on a domain $\Omega \subset \mathbb{M}^{2}$, with positive constant extrinsic curvature $K$ and whose boundary is contained in the slice $\mathbb{M}^{2} \times\{0\}$. Let $k$ be the minimum of the Gauss curvature on $\Omega \subset \mathbb{M}^{2}$. Then, there exists a constant $c_{K}$ (depending only on $K$ and $k$ ) such that $|h(p)| \leq c_{K}$ for all $p \in S$ ( $h$ is the height function on the graph).

Also, horizontal height (or distance) estimates are obtained,

Theorem 6.2. Let $S$ be a compact embedded surface in $\mathbb{H}^{2} \times \mathbb{R}$, with extrinsic curvature a constant $K>0$. Let $P$ be a vertical plane in $\mathbb{H}^{2} \times \mathbb{R}$ and assume that $\partial S \subset P$. Then the distance from $S$ to $P$ is bounded; i.e., there is a constant $d$, independent of $S$, such that

$$
\operatorname{dist}(q, P) \leq d \quad \text { for all } q \in S .
$$

We remark that the proof of this result works for $H$-surfaces in $\mathbb{H}^{2} \times \mathbb{R}$ with $H>1 / 2$, thus this result, together with the vertical height estimates given in [AEG2] for $H$-surfaces with $H>1 / 2$, generalizes Theorem 1.1 in [NR] for $H$-surfaces with $H>1 / 2$. More precisely,

Theorem 7.2. For $K>0$ (or $H>1 / 2$ ) there is no properly embedded $K$-surface (H-surface) in $\mathbb{H}^{2} \times \mathbb{R}$, with finite topology and one end.

Finally, in Section 7 we classify the complete immersed $K$-surfaces in $\mathbb{H}^{2} \times \mathbb{R}$ and $\mathbb{S}^{2} \times \mathbb{R}$. 
Theorem 7.3. The complete immersions with positive constant extrinsic curvature $K$ in $\mathbb{H}^{2} \times \mathbb{R}$ and $\mathbb{S}^{2} \times \mathbb{R}$ are the rotational spheres given in Section 5 .

\section{Notation}

In Sections 2, 3 and 4 we will use the Poincaré disk model of $\mathbb{H}^{2}$. In Section 5 we will work in the hyperboloid of one sheet model of $\mathbb{H}^{2}$ in the Lorentz-Minkowski 3 -space $\mathbb{L}^{3}$. We make this precise in Section 5.

In the Poincaré model, $\mathbb{H}^{2}$ is represented as the domain

$$
\mathbb{D}=\left\{z \equiv(x, y) \in \mathbb{R}^{2}:|z|^{2}=x^{2}+y^{2}<1\right\}
$$

endowed with the metric $g_{-1}=\frac{4|d z|^{2}}{\left(1-|z|^{2}\right)^{2}}$.

The complete geodesics in this model are given by arcs of circles or straight lines which are orthogonal to the boundary at infinity

$$
\mathbb{S}_{\infty}^{1}=\left\{z \in \mathbb{R}^{2}:|z|=1\right\} .
$$

Thus, the asymptotic boundary of a set $\Omega \subset \mathbb{H}^{2}$ is

$$
\partial_{\infty} \Omega=\operatorname{cl}(\Omega) \cap \mathbb{S}_{\infty}^{1},
$$

where $\operatorname{cl}(\Omega)$ is the closure of $\Omega$ in $\left\{z \in \mathbb{R}^{2}:|z| \leq 1\right\}$.

We orient $\mathbb{H}^{2}$ so that its boundary at infinity is oriented counter-clockwise. Let $\gamma$ be a complete oriented geodesic in $\mathbb{H}^{2}$, then

$$
\partial_{\infty} \gamma=\left\{\gamma^{-}, \gamma^{+}\right\}
$$

where $\gamma^{-}=\lim _{t \rightarrow-\infty} \gamma(t)$ and $\gamma^{+}=\lim _{t \rightarrow+\infty} \gamma(t)$. Here $t$ is the arc length along $\gamma$. We will often identify a geodesic $\gamma$ with its boundary at infinity, writing

$$
\gamma=\left\{\gamma^{-}, \gamma^{+}\right\}
$$

Definition 2.1. Let $\theta_{1}, \theta_{2} \in \mathbb{S}_{\infty}^{1}$. We define the oriented geodesic joining $\theta_{1}$ and $\theta_{2}$, $\gamma\left(\theta_{1}, \theta_{2}\right)$, as the oriented geodesic from $\theta_{1} \in \mathbb{S}_{\infty}^{1}$ to $\theta_{2} \in \mathbb{S}_{\infty}^{1}$. Here we represent points on the circle as real numbers (angles) by their image under the exponential map.

We observe that given an oriented geodesic $\gamma=\left\{\gamma^{-}, \gamma^{+}\right\}$in $\mathbb{H}^{2}$, then $\mathbb{H}^{2} \backslash \gamma$ has two connected components. We will distinguish them using the following notation,

Definition 2.2. Let $J$ be the standard counter-clockwise rotation operator. We call exterior set of $\gamma$ in $\mathbb{H}^{2}$, ext $\mathbb{H}^{2}(\gamma)$, the connected component of $\mathbb{H}^{2} \backslash \gamma$ towards which $J \gamma^{\prime}$ points. The other connected component of $\mathbb{H}^{2} \backslash \gamma$ is called the interior set of $\gamma$ in $\mathbb{H}^{2}$ and denoted by $\operatorname{int}_{\mathbb{H}^{2}}(\gamma)$. 
On the other hand, we consider the product space $\mathbb{H}^{2} \times \mathbb{R}$ represented as the domain

$$
\mathbb{H}^{2} \times \mathbb{R}=\left\{(x, y, t) \in \mathbb{R}^{3}: x^{2}+y^{2}<1\right\}
$$

endowed with the product metric $\langle\rangle=,g_{-1}+d t^{2}$. In addition, we denote by $\pi: \mathbb{H}^{2} \times \mathbb{R} \rightarrow \mathbb{H}^{2} \times\{0\}$ the usual projection and $\frac{\partial}{\partial t}$ the gradient of the function $t$ in $\mathbb{H}^{2} \times \mathbb{R}$.

Given a complete oriented geodesic $\gamma$ in $\mathbb{H}^{2} \times\{0\}$, we will call $\gamma \times \mathbb{R}$ a vertical plane of $\mathbb{H}^{2} \times \mathbb{R}$ and we will call a slice $\mathbb{H}^{2} \times\{\tau\}$ a horizontal plane. Note that a vertical plane is isometric to $\mathbb{R}^{2}$ and a horizontal plane is isometric to $\mathbb{H}^{2}$.

The notions of the interior and exterior domains of a horizontal oriented geodesic extend naturally to vertical planes.

Definition 2.3. For a complete oriented geodesic $\gamma$ in $\mathbb{H}^{2} \times\{0\} \equiv \mathbb{H}^{2}$ we call, respectively, interior and exterior of the vertical plane $P=\gamma \times \mathbb{R}$ the sets

$$
\operatorname{int}_{\mathbb{H}^{2} \times \mathbb{R}}(P)=\operatorname{int}_{\mathbb{H}^{2}}(\gamma) \times \mathbb{R} \text { and } \operatorname{ext}_{\mathbb{H}^{2} \times \mathbb{R}}(P)=\operatorname{ext}_{\mathbb{H}^{2}}(\gamma) \times \mathbb{R} \text {. }
$$

We will often use foliations by vertical planes of $\mathbb{H}^{2} \times \mathbb{R}$. We now make this precise.

Definition 2.4. Let $P$ be a vertical plane in $\mathbb{H}^{2} \times \mathbb{R}$ and let $\gamma(t)$ be an oriented horizontal geodesic in $\mathbb{H}^{2} \times\{0\}$, with $t$ being the arc length along $\gamma, \gamma(0)=p_{0} \in P$, $\gamma^{\prime}(0)$ orthogonal to $P$ at $p_{0}$ and $\gamma(t) \in \operatorname{ext}_{\mathbb{H}^{2} \times \mathbb{R}}(P)$ for $t>0$. We define the oriented foliation of vertical planes along $\gamma$, denoted by $P_{\gamma}(t)$, to be the vertical planes orthogonal to $\gamma(t)$ with $P=P_{\gamma}(0)$.

To finish, we will give the definition of a particular type of curve in a vertical plane.

Definition 2.5. Let $P$ be a vertical plane and let $\alpha$ be a complete embedded convex curve in $P$. We say that $\alpha$ is vertical (in $P$ ) if there exist a point $p \in \alpha$, called a vertical point, and a vertical direction $v= \pm \frac{\partial}{\partial t}$, such that the half-line $p+s v, s>0$, is contained in the convex body bounded by $\alpha$ in $P$ (cf. Figure 1).

\section{A Hadamard-Stoker type theorem}

This section is devoted to the proof of a Hadamard-Stoker type theorem in $\mathbb{H}^{2} \times \mathbb{R}$. Let us consider a surface $S$ and $\psi: S \rightarrow \mathbb{H}^{2} \times \mathbb{R}$ an immersion with positive extrinsic curvature $K=\operatorname{det}(\mathrm{II}) / \operatorname{det}(\mathrm{I})$, where I and II are the first and second fundamental forms of $S$. 


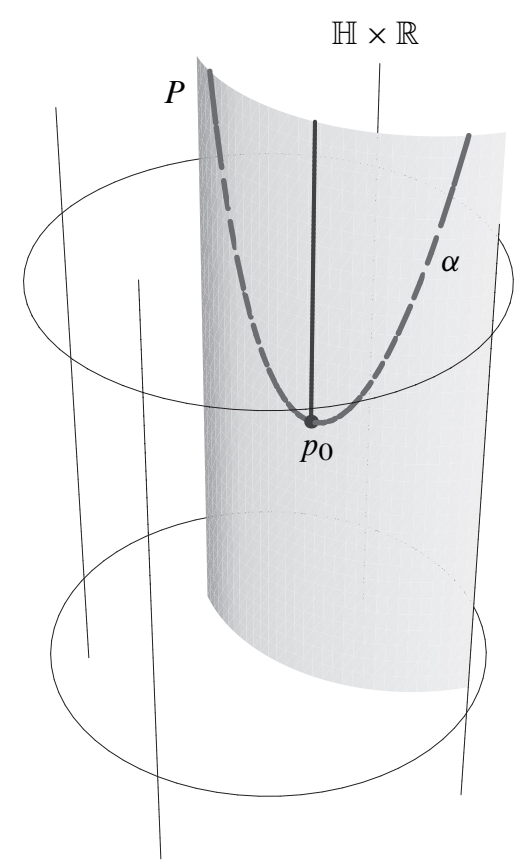

Figure 1. Definition of vertical point.

Observe that the definition of $K$ does not depend on the local choice of a unit normal vector field $N$. Nevertheless, $N$ can be globally chosen since $K>0$, that is, II is definite. From now on we will identify $\psi(S)$ with $S$.

We begin with an elementary, but useful, result.

Proposition 3.1. Let $S$ be an immersed surface with positive extrinsic curvature in $\mathbb{H}^{2} \times \mathbb{R}$. Let $P$ be either a horizontal or a vertical plane in $\mathbb{H}^{2} \times \mathbb{R}$. If $S$ and $P$ intersect transversally then each connected component $C$ of $S \cap P$ is a strictly convex curve in $P$.

Proof. Let us parametrize $C$ as $\alpha(t)$, where $t$ is the $\operatorname{arc}$ length. Then since $P$ is a totally geodesic plane, we have

$$
\nabla_{\alpha^{\prime}}^{P} \alpha^{\prime}=\bar{\nabla}_{\alpha^{\prime}} \alpha^{\prime}=\nabla_{\alpha^{\prime}}^{S} \alpha^{\prime}+\operatorname{II}\left(\alpha^{\prime}, \alpha^{\prime}\right) N
$$

where $\nabla^{P}, \bar{\nabla}$ and $\nabla^{S}$ are the connections on $P, \mathbb{H}^{2} \times \mathbb{R}$ and $S$ respectively. Since the extrinsic curvature is positive we have $\operatorname{II}\left(\alpha^{\prime}, \alpha^{\prime}\right) \neq 0$. Thus $\nabla_{\alpha^{\prime}}^{P} \alpha^{\prime} \neq 0$, that is, the geodesic curvature of $C$ vanishes nowhere on $P$. 
Definition 3.1. Let $S \subset \mathbb{H}^{2} \times \mathbb{R}$ be a surface. We say that $S$ has a simple end if the boundary at infinity of $\pi(S) \subset \mathbb{H}^{2} \times\{0\} \equiv \mathbb{H}^{2}$ is a unique point $\theta_{0} \in \mathbb{S}_{\infty}^{1}$ and, in addition, for all $\theta_{1}, \theta_{2} \in \mathbb{S}_{\infty}^{1} \backslash\left\{\theta_{0}\right\}$ the intersection of the vertical plane $\gamma\left(\theta_{1}, \theta_{2}\right) \times \mathbb{R}$ and $S$ is empty or a compact set.

Now, we can establish the main theorem of this section.

Theorem 3.1. Let $S$ be a complete connected immersed surface in $\mathbb{H}^{2} \times \mathbb{R}$ with $K>0$. Then $S$ must be properly embedded and bounds a strictly convex domain in $\mathbb{H}^{2} \times \mathbb{R}$. Moreover, $S$ is homeomorphic to $\mathbb{S}^{2}$ or $\mathbb{R}^{2}$. In the latter case, $S$ is a graph over a convex domain of $\mathbb{H}^{2} \times\{0\}$ or $S$ has a simple end.

Proof. We first distinguish two cases, depending on the existence of a point on $S$ with horizontal unit normal, or equivalently, depending on the existence of a vertical tangent plane.

Suppose there is no point $p \in S$ with a vertical tangent plane at $p$. We will show that $S$ is a graph and homeomorphic to $\mathbb{R}^{2}$.

Let $P$ be a vertical plane which meets $S$ transversally. Let $\gamma$ be an oriented horizontal geodesic orthogonal to $P$ and consider the foliation $P_{\gamma}(t)$ of vertical planes along $\gamma$ (see Definition 2.4). Now, if $P_{\gamma}(t) \cap S \neq \emptyset$, using that there is no point $p \in S$ with a vertical tangent plane at $p$ and Proposition 3.1, each connected component of $P_{\gamma}(t) \cap S$ is a non-compact complete embedded strictly convex curve. Otherwise, if a connected component has a self-intersection or it is compact, then it has a point with a vertical tangent line, which means that $S$ has a point with a vertical tangent plane at that point (cf. Figure 2).

Let $C(0)$ be an embedded component of $P \cap S=P_{\gamma}(0) \cap S$. Let us consider how $C(0)$ varies as $t$ increases to $+\infty$. No two points of $P_{\gamma}\left(t_{0}\right) \cap S$ can join at some $t_{0}>0$, since this would produce a vertical tangent plane at some point. So the component $C(0)$ of $P_{\gamma}(0)$ varies continuously to one embedded curve $C(t)$ of $P_{\gamma}(t) \cap S$ as $t$ increases. The only change possible is that $C(t)$ goes to infinity as $t$ converges to some $t_{1}$ and disappears in $P_{\gamma}\left(t_{1}\right)$.

Similarly $C(0)$ varies continuously to one embedded curve of $P_{\gamma}(t) \cap S$ as $t \rightarrow-\infty$. Hence $S$ connected yields $P_{\gamma}(t) \cap S$ is at most one component for all $t$. So, we conclude that $S$ is a vertical graph. To finish, we observe that $P_{\gamma}(t) \cap S$ is empty or homeomorphic to $\mathbb{R}$ for each $t$, hence $S$ is topologically $\mathbb{R}^{2}$.

Now, for the rest of the proof we suppose that there is a point $p_{0} \in S$ with a vertical plane $P$ tangent at $p_{0}$. We will show that $S$ is homeomorphic to $\mathbb{S}^{2}$ or $S$ is homeomorphic to $\mathbb{R}^{2}$ and has a simple end.

By assumption, it is easy to see that there exist neighborhoods $p_{0} \in U \subset S$ and $V \subset P$ such that $U$ is a horizontal graph over $V$. Also, because of $K\left(p_{0}\right)>0, S$ is strictly locally convex at $p_{0}$, hence we can assume that $U \subset S$ is on one side of $P$ 


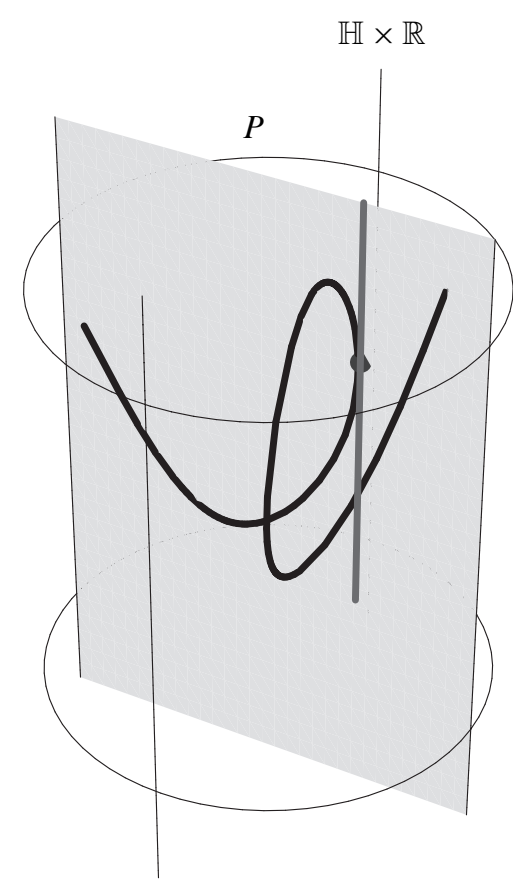

Figure 2. No self-intersection.

(cf. Figure 3). Let $P_{\gamma}(t)$ be the foliation of vertical planes along $\gamma, \gamma$ a horizontal geodesic with $\gamma(0)=p_{0}$ and $\gamma^{\prime}(0)$ orthogonal to $P$. Note that, up to an isometry, we can suppose that $U \backslash\left\{p_{0}\right\} \subset \operatorname{ext}_{\mathbb{H}^{2} \times \mathbb{R}}(P), p_{0} \in \mathbb{H}^{2} \times\{0\}$ and $\gamma=\{\pi / 2,3 \pi / 2\}$.

From Proposition 3.1 and the fact that locally $S$ is a graph, there exist $\varepsilon>0$ such that $P_{\gamma}(t) \cap U$ are embedded compact strictly convex curves for all $0<t<\varepsilon$. For $0<t<\varepsilon$, let $C(t)$ denote the connected component of $P_{\gamma}(t) \cap S$ which coincides with $P_{\gamma}(t) \cap U$ (cf. Figure 4). Perhaps $P_{\gamma}(t) \cap S$ has other components distinct from $C(t)$ for each $0<t<\varepsilon$, but we only care how $C(t)$ varies as $t$ increases. We also denote by $C(t)$ the continuous variation of the curves $P_{\gamma}(t) \cap S$, when $t>\varepsilon$.

We distinguish two cases:

A. $C(t)$ remains compact as $t$ increases.

By topological arguments it is easy to show that, if $C(t)$ remains compact and non-empty as $t$ increases, then the $C(t)$ are embedded compact strictly convex curves or a point.

A.1. If $C(t)$ remains compact and non-empty as $t \rightarrow+\infty$, then since $S$ is connected, $S$ must be embedded. In addition, because $C(0)$ is a point and $C(t)$ is 


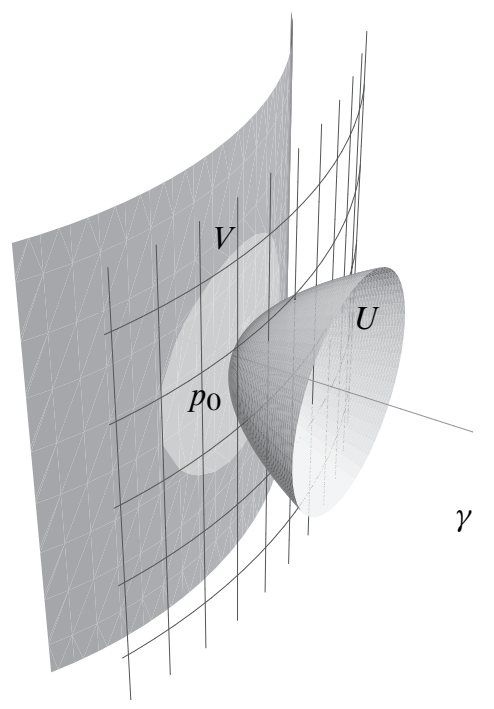

Figure 3. $S$ is strictly locally convex at $p_{0}$.
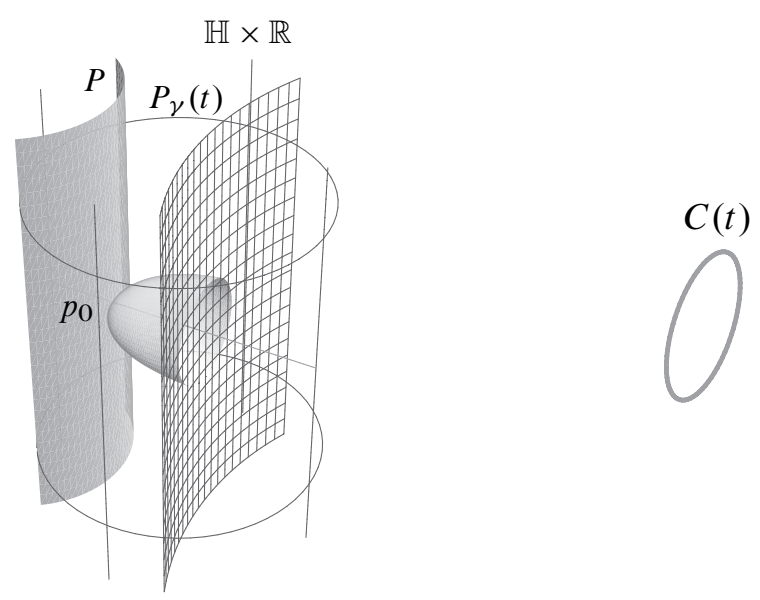

Figure 4. $C(t)$ are embedded compact strictly convex curves for all $0<t<\varepsilon$.

homeomorphic to a circle for every positive $t, S$ is homeomorphic to $\mathbb{R}^{2}$. Now, from the fact that $C(t)$ remains compact, then

$$
\partial_{\infty} \pi(S)=\{3 \pi / 2\} \subset \mathbb{S}_{\infty}^{1}
$$

and $S$ has a simple end (cf. Figure 5). 

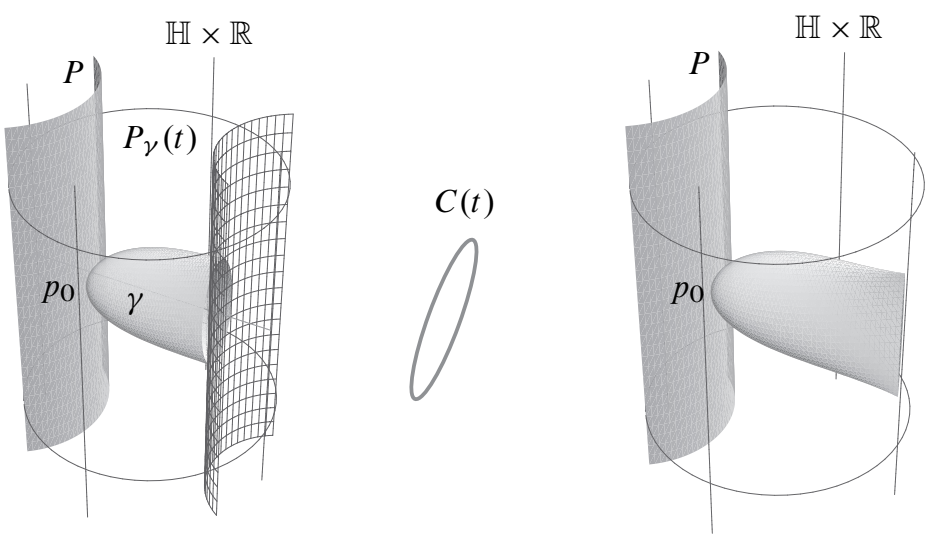

Figure 5. $C(t)$ remains compact as $t$ increases.

A.2. If there exists $\bar{t}>0$ such that $C(t)$ is compact for all $0<t<\bar{t}$ and the component $C(t)$ disappears for $t>\bar{t}$, then, using that $S$ is connected, $S$ is either compact, embedded and topologically $\mathbb{S}^{2}$, or non compact, embedded and topologically $\mathbb{R}^{2}$. That is, if $C(t)$ converges to a compact set as $t$ converges to $\bar{t}$, then $C(\bar{t})$ must be a point (because our surface has no boundary) and $S$ is a sphere (cf. Figure 6). Otherwise the $C(t)$ drift off to infinity as $t$ converges to $\bar{t}$ and $S$ is topologically a plane.
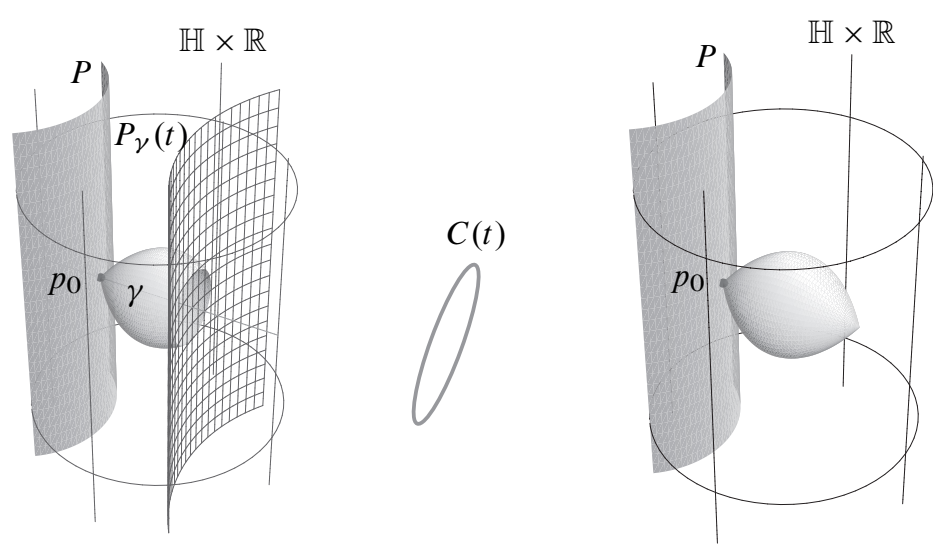

Figure 6. $C(t)$ goes to a point.

We now show that in the latter case, the vertical projection $\pi$ of $S$ has asymptotic boundary one of the two points at infinity of $\pi\left(P_{\gamma}(\bar{t})\right)$. 
Without lost of generality we can assume that $P_{\gamma}(\bar{t})=\beta \times \mathbb{R}$ where $\beta=$ $\left\{\beta^{-}, \beta^{+}\right\}$. Consider the vertical plane $Q=\gamma \times \mathbb{R}$. Let $\widetilde{C}$ be the component of $Q \cap S$ containing $p_{0}$. First observe that $\widetilde{C}$ is compact, otherwise it would intersect the line $Q \cap P_{\gamma}(\bar{t})$ in two points, which is not the case. Thus, we can consider the disk $\widetilde{D}$ bounded by $\widetilde{C}$ on $S$.

Let $Q_{\beta}(t)$ denote the foliation by vertical planes along $\beta, Q_{\beta}(0)=Q$. There exists $t_{0}$ (we can assume $t_{0}<0$ ) satisfying $Q_{\beta}\left(t_{0}\right)$ touches $\widetilde{D}$ on one side of $\widetilde{D}$ by compactness. Let $q_{0} \in \widetilde{D} \cap Q_{\beta}\left(t_{0}\right)$ be the point where they touch. Consider the variation $\widetilde{C}(t)$ of $q_{0}$ on $S \cap Q_{\beta}(t)$ from $t=t_{0}$ to infinity. Then, $\widetilde{C}(t)$ is a convex embedded curve for $t$ in a maximal interval $\left(t_{0}, \bar{t}_{0}\right)$ with $0<\bar{t}_{0} \leq \infty$. Hence, $S$ is foliated by the $\widetilde{C}(t), \widetilde{C}=\widetilde{C}(0)=Q \cap S$ and $\beta^{-} \notin \partial_{\infty} \pi(S)$ because $S$ is on one side of $Q_{\beta}\left(t_{0}\right)$.

Now, we will show that $\partial_{\infty} \pi(S)=\left\{\beta^{+}\right\}$. Let $\gamma(\theta)$ denote the complete horizontal geodesic starting at $p_{0}$ and making an angle $\theta$ with $\gamma$ at $p_{0}$. Assume $\gamma(\theta)$ enters the side of $Q$ containing $\beta^{+}$, for $0<\theta<\pi / 2$. Let $\bar{\theta}$ be the value of $\theta$ such that $\gamma(\bar{\theta})$ is asymptotic to $\beta^{+}$. Let $Q(\theta)=\gamma(\theta) \times \mathbb{R}$. For each $\theta, 0 \leq \theta<\bar{\theta}$, we have $S \cap Q(\theta)$ is one connected embedded compact curve $C^{\prime}(\theta)$. The proof of this is the same as the previous one for $\widetilde{C}$. Notice that each $C^{\prime}(\theta)$ is non empty, since $p_{0} \in C^{\prime}(\theta)$.

Now $C^{\prime}(\bar{\theta})$ can not be compact, otherwise $S$ could not be asymptotic to the plane $P_{\gamma}(\bar{t})$, a contradiction.

In order to complete the proof of the Case A.2 we show that $S$ has a simple end. Observe that $C^{\prime}(\theta)$ is compact, $\bar{\theta}<\theta<\pi / 2$ because $S=\bigcup_{0 \leq t<\bar{t}} C(t)$. Moreover, $C^{\prime}(\theta) \subset \widetilde{D},-\pi / 2<\theta<0$, and $\widetilde{D}$ is compact. Thus, it is easy to conclude that $S$ has a simple end.

Thus we have proved that in Case A.2, $S$ is either a properly embedded sphere or $S$ is a properly embedded plane with a simple end at $\beta^{+}$(cf. Figure 5, picture on the right).

B. $C(t)$ becomes non-compact.

Let $\bar{t}>0$ be the smallest $t$ with $C(\bar{t})$ non-compact, $C(\bar{t})$ the limit of the $C(t)$ as $t \rightarrow \bar{t}, C(\bar{t})$ is an embedded strictly convex curve in $P_{\gamma}(\bar{t})$.

Claim 1. We now show that $C(\bar{t})$ is not vertical (see Definition 2.5).

Let us assume that $C(\bar{t})$ is vertical, and let $q \in C(\bar{t})$ be a vertical point (cf. Figure 7). First of all, note that $\widetilde{S}=\bigcup_{0 \leq t \leq \bar{t}} C(t) \subset S$ is embedded. Let $q^{\prime}=\pi(q) \in \mathbb{H}^{2} \times\{0\}$ and let us consider $\Gamma_{p_{0} q^{\prime}}$ the complete horizontal geodesic joining $p_{0}$ and $q^{\prime}$. Let $Q=\Gamma_{p_{0} q^{\prime}} \times \mathbb{R}$, and consider $r_{0}=Q \cap P_{\gamma}(0)$ and $r_{\bar{t}}=Q \cap P_{\gamma}(\bar{t})$. Note that $r_{0}$ and $r_{\bar{t}}$ are parallel lines in $Q$. Also, 

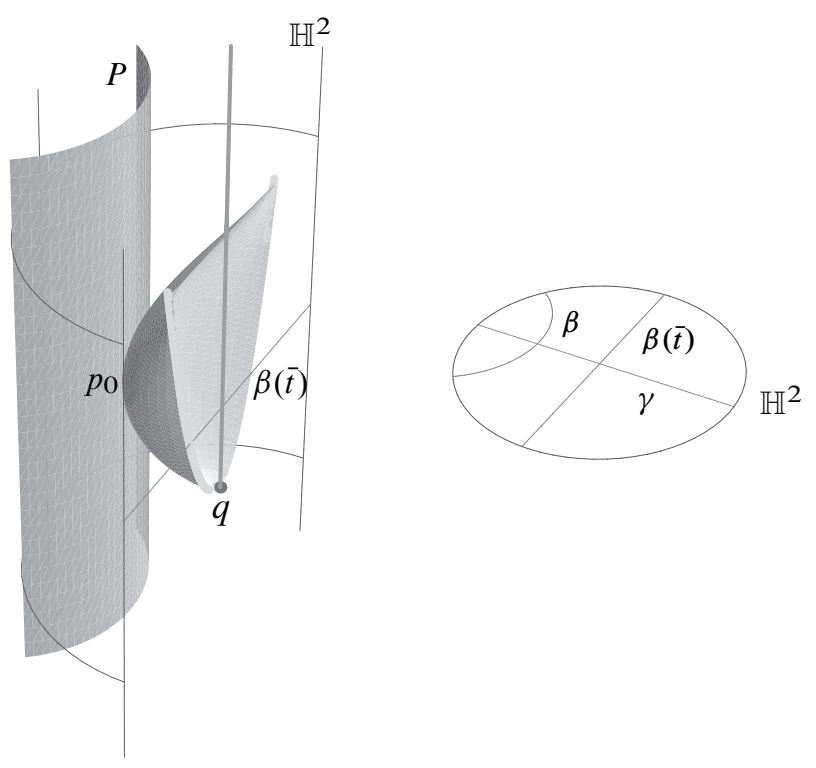

Figure 7. $C(\bar{t})$ becomes vertical.

$\alpha_{Q}=Q \cap \widetilde{S}$ is a non-compact embedded strictly convex curve in $Q$ such that $r_{0}$ is tangent to $\alpha_{Q}$ at $p_{0} \in \alpha_{Q}$ and $\alpha_{Q} \cap r_{\bar{t}}$ is exactly one point, since $C(\bar{t})$ is vertical. But this is a contradiction because $\alpha_{Q}$ is a strictly convex curve in $Q$, which is isometric to $\mathbb{R}^{2}$, and it must intersect $r_{\bar{t}}$ twice.

Thus, $C(\bar{t})$ is not vertical, and we claim that

Claim 2. $\partial_{\infty} \pi(C(\bar{t}))$ is one point.

Let us denote by $D(t)$ the convex body bounded by $C(t)$ in $P_{\gamma}(t)$ for each $0<t<\bar{t}$. Thus, the limit, $D(\bar{t})$, of $D(t)$ as $t$ increases to $\bar{t}$ is an open convex body bounded by $C(\bar{t})$ in $P_{\gamma}(\bar{t})$, which is isometrically $\mathbb{R}^{2}$. If $\partial_{\infty} \pi(C(\bar{t}))$ has two points, the only possibility is that $C(\bar{t})$ is vertical, which is impossible by Claim 1 (cf. Figure 7)

Let $\delta_{0}>0$ and $t_{\delta_{0}}<\bar{t}$ such that $P\left(t_{\delta_{0}}\right)=\Gamma\left(\delta_{0}\right) \times \mathbb{R}$ where $\Gamma\left(\delta_{0}\right)=$ $\left\{\delta_{0}, \pi-\delta_{0}\right\}$. We denote by $\widetilde{S}_{1}=\bigcup_{0 \leq t \leq t_{\delta_{0}}} C(t) \subset S$ and note that $\widetilde{S}_{1}$ is connected and embedded.

Let us consider the complete horizontal geodesic given by

$$
\Gamma\left(\delta_{0}, s\right)=\left\{\delta_{0}, \pi-\delta_{0}+s\right\}
$$

and the vertical plane $Q(s)=\Gamma\left(\delta_{0}, s\right) \times \mathbb{R}$, for each $s \geq 0$. So, $Q(0)=P_{\gamma}\left(t_{\delta_{0}}\right)$ 
and $Q(0) \cap \widetilde{S}_{1}=C\left(t_{\delta_{0}}\right)$ is an embedded compact strictly convex curve. Let us consider how $\alpha(s)=Q(s) \cap S$ varies as $s$ increases to $\pi+\delta_{0}$. At this point, we have two cases:

B.1. $\alpha(s)$ remains compact for all $0 \leq s<\pi+\delta_{0}$.

This case, letting $\delta_{0} \rightarrow 0$, corresponds to Case A.1 (cf. Figure 8).
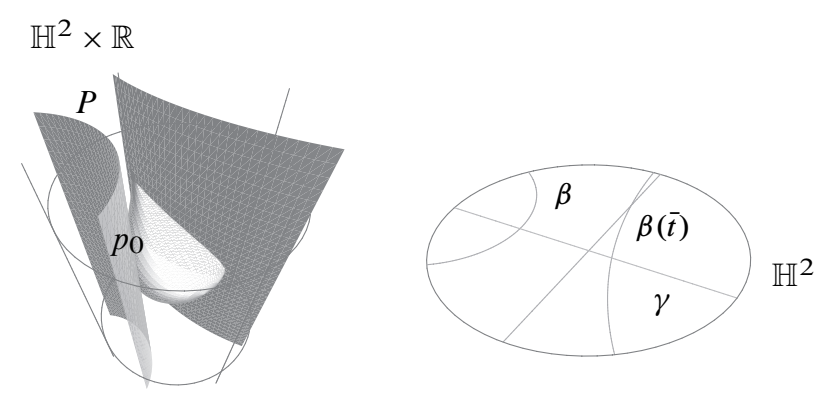

Figure 8. $\alpha(s)$ remains compact.

So, without lost of generality we can assume that $P_{\gamma}(\bar{t})=\Gamma(\bar{t}) \times \mathbb{R}$ where $\Gamma(\bar{t})=\{0, \pi\}$ and (cf. Figure 9)

$$
\partial_{\infty} \pi(C(\bar{t}))=\{0\} .
$$
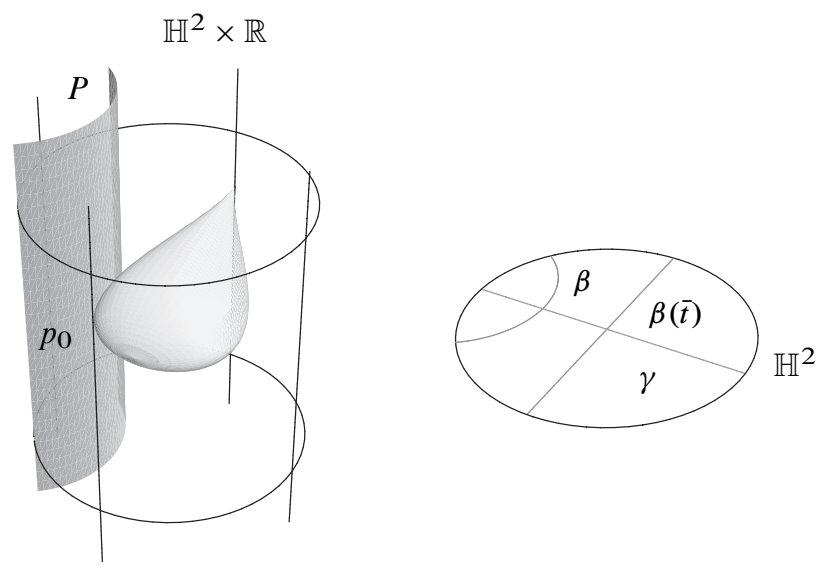

Figure 9. $S$ has a simple end. 
B.2. $\alpha(s)$ becomes non-compact.

Let $0<\bar{s}<\pi+\delta_{0}$ be the smallest $s$ with $\alpha(\bar{s})$ non-compact, $\alpha(\bar{s})$ is the limit of the $\alpha(s)$ as $s \rightarrow \bar{s}$. So, without lost of generality we can assume that $P_{\gamma}(\bar{t})=\Gamma(\bar{t}) \times \mathbb{R}$ where $\Gamma(\bar{t})=\{0, \pi\}$ and

$$
\partial_{\infty} \pi(C(\bar{t}))=\{0\} .
$$

Also,

$$
\partial_{\infty} \pi(\alpha(\bar{s}))=\left\{\pi-\delta_{0}+\bar{s}\right\},
$$

otherwise it must be $\left\{\delta_{0}\right\}$ which contradicts (3.2).

Clearly $\delta_{0}<\bar{s}$. For each $\delta \leq \delta_{0}$ we consider the complete horizontal geodesic given by $\sigma(\delta)=\left\{\delta, \pi+\bar{s}-\delta_{0}-\delta\right\}$ and the vertical plane $T(\delta)=\sigma(\delta) \times \mathbb{R}$. Let us denote by $\widetilde{S}_{2}=\bigcup_{0 \leq s \leq \bar{s}-2 \delta_{0}} \alpha(s) \subset S$ and note that $\widetilde{S}_{2}$ is connected and embedded, so, $\widetilde{S}=\widetilde{S}_{1} \cup \widetilde{\widetilde{S}}_{2} \subset S$ is connected and embedded. For each $\delta$, $0<\delta \leq \delta_{0}, E(\delta)=T(\delta) \cap \widetilde{S}$ is a strictly convex compact embedded curve in $T(\delta)$. As $\delta \rightarrow 0$, these curves converge to a convex curve in $T(0)$ with $\partial_{\infty} \pi(E(0))$ the two points $\left\{0, \pi-\delta_{0}+\bar{s}\right\}$. This contradicts Claim 2. Hence $\alpha(s)$ can not become non-compact and we are in the Case B.1.

Finally, $S$ bounds a strictly convex body follows from Proposition 3.1, and the fact that every geodesic in $\mathbb{H}^{2} \times \mathbb{R}$ lies in a vertical plane, i.e., let $p$ and $q$ be two points in the domain $W$ of $\mathbb{H}^{2} \times \mathbb{R}$ bounded by $\Sigma$, such that the mean curvature vector of $\Sigma$ points into this domain $W$. Let $P$ be a vertical plane containing $p$ and $q$. The intersection of $P$ with $\Sigma$ is convex in $P$, hence the geodesic of $P$ (which is also an ambient geodesic), is contained in $W$. Thus $W$ is convex.

This completes the proof of Theorem 3.1.

\section{Complete surfaces in $\mathbb{H}^{2} \times \mathbb{R}$ with $K>0$ and a simple end}

This section is devoted to the construction of some examples of complete embedded surfaces with positive extrinsic curvature and a simple end.

Note that if $S$ has a simple end, then for each $t \in \mathbb{R}$ where $S \cap\left(\mathbb{H}^{2} \times\{t\}\right)$ is not compact, the intersection is a convex curve in $\mathbb{H}^{2} \times\{t\}$ with asymptotic boundary one point. Also, the point at infinity of each horizontal section is the same, that is, $\partial_{\infty} \pi\left(S \cap \mathbb{H}^{2} \times\{t\}\right)=\{\bar{\theta}\} \in \mathbb{S}_{\infty}^{1}$.

Bearing this in mind, we fix a point $\bar{\theta} \in \mathbb{S}_{\infty}^{1}$, say $\bar{\theta}=0 \equiv(1,0)$, and consider the 1-parameter isometry group given by

$$
\begin{aligned}
& F_{t}(x, y, z) \\
& \quad=\left(1+\frac{4(x-1)}{4+4 t y+t^{2}\left((x-1)^{2}+y^{2}\right)}, \frac{4 y+2 t\left((x-1)^{2}+y^{2}\right)}{4+4 t y+t^{2}\left((x-1)^{2}+y^{2}\right)}, z\right) .
\end{aligned}
$$


Here, the orbit of any point $p \in \mathbb{H}^{2} \times \mathbb{R}$ is a horocycle $H_{p}$ contained in a slice such that $\partial_{\infty} \pi\left(H_{p}\right)=\{\bar{\theta}\}$.

Let $P$ be a plane orthogonal to every orbit, say $P=\left\{(x, y, z) \in \mathbb{H}^{2} \times \mathbb{R}: y=0\right\}$. We parametrize $P$ as

$$
\psi(x, y)=\left(\frac{e^{x}-1}{e^{x}+1}, 0, y\right)
$$

in such a way that its induced metric is $d x^{2}+d y^{2}$.

Now, let $\alpha(y)=\psi(\rho(y), y)$ be a curve in the vertical plane $P$ for a suitable function $\rho$ and consider the helicoidal surface $S_{\rho}$ given by $f_{\rho}(y, t)=F_{t}(\alpha(y))$.

Then, it is easy to check that the extrinsic curvature of $S_{\rho}$ is

$$
K=\frac{\rho^{\prime \prime}(y)}{\left(1+\rho^{\prime}(y)^{2}\right)^{2}} \text {. }
$$

Thus, we obtain

Proposition 4.1. Let $d=\left(y_{1}, y_{2}\right)$ be an open interval where $-\infty \leq y_{1}<y_{2} \leq$ $+\infty$, and $\rho: \mathcal{d} \rightarrow \mathbb{R}$ a function such that $\rho^{\prime \prime}(y)>0$ for all $y \in d, \lim _{y \rightarrow y_{1}} \rho(y)=$ $\lim _{y \rightarrow y_{2}} \rho(y)=+\infty$. Then the surface $S_{\rho}$ is a properly embedded surface with positive extrinsic curvature and a simple end (cf. Figure 10).

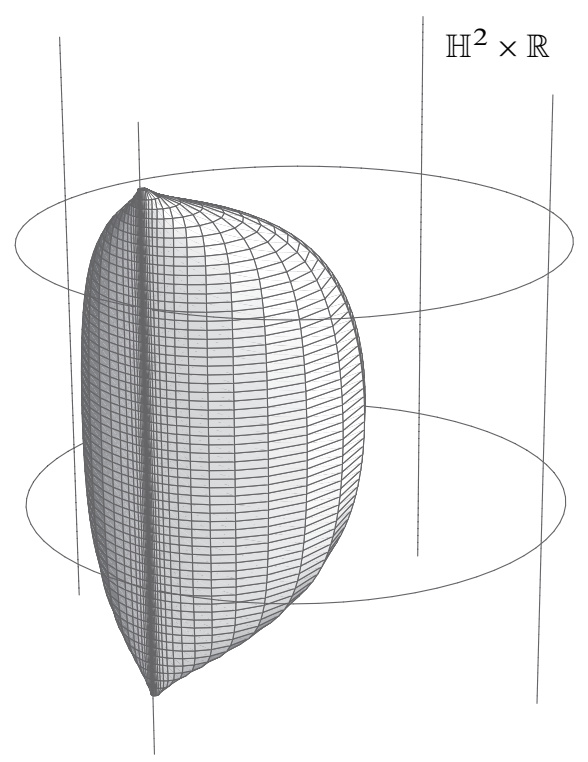

Figure 10. Example of a surface with a simple end. 


\section{Complete revolution surfaces of constant positive extrinsic curvature}

In this section we focus our attention on the study of the complete revolution surfaces of positive constant extrinsic curvature in $\mathbb{H}^{2} \times \mathbb{R}$.

Let us consider the Lorentz-Minkowski 4-space $\mathbb{L}^{4}$ with induced metric $-d x_{1}^{2}+$ $d x_{2}^{2}+d x_{3}^{2}+d x_{4}^{2}$. Here, we consider $\mathbb{H}^{2} \times \mathbb{R}$ as the submanifold of $\mathbb{L}^{4}$ given by

$$
\mathbb{H}^{2} \times \mathbb{R}=\left\{\left(x_{1}, x_{2}, x_{3}, x_{4}\right) \in \mathbb{L}^{4}:-x_{1}^{2}+x_{2}^{2}+x_{3}^{2}=-1, x_{1}>0\right\} .
$$

It is well known that the special orthogonal group $\mathrm{SO}(2)$ can be identified with the subgroup of isometries of $\mathbb{H}^{2} \times \mathbb{R}$ (rotations) which preserves the orientation and fixes an axis $\{p\} \times \mathbb{R}$, with $p \in \mathbb{H}^{2} \times\{0\}$.

Up to isometries, we can assume that the axis is given by $\{(1,0,0)\} \times \mathbb{R}$. In this case, the set $P=\left\{\left(x_{1}, x_{2}, x_{3}, x_{4}\right) \in \mathbb{H}^{2} \times \mathbb{R}: x_{2} \geq 0, x_{3}=0\right\}$ intersects every $\mathrm{SO}(2)$-orbit once. Thus, every revolution surface with that axis can be obtained under the $\mathrm{SO}(2)$-action of a curve on $P$.

Let $\alpha(t)=(\cosh k(t), \sinh k(t), 0, h(t)) \subset P$, where $k(t) \geq 0$ and $t$ the arc length of $\alpha$, that is, $k^{\prime}(t)^{2}+h^{\prime}(t)^{2}=1$. If $\alpha(t)$ generates a complete surface with positive extrinsic curvature then, from Theorem 3.1, this revolution surface $S$ must be topologically a sphere or a graph on an open domain in $\mathbb{H}^{2}$, and so $\alpha(t)$ intersects the axis at least once. In fact, the curve intersects the axis orthogonally; otherwise the revolution surface would not be smooth. But, from the vertical height estimates of Section 6, there is no complete revolution $K$-surface with one end.

Now, $S$ can be parametrized by

$$
\psi(t, v)=(\cosh k(t), \sinh k(t) \cos v, \sinh k(t) \sin v, h(t)) .
$$

A straightforward computation shows that the principal curvatures of $S$ are given by

$$
\begin{aligned}
& \lambda_{1}=k^{\prime} h^{\prime \prime}-k^{\prime \prime} h^{\prime}, \\
& \lambda_{2}=h^{\prime} \operatorname{coth} k,
\end{aligned}
$$

and so its extrinsic curvature is

$$
K=h^{\prime} \operatorname{coth} k\left(k^{\prime} h^{\prime \prime}-k^{\prime \prime} h^{\prime}\right),
$$

where ' denotes the derivative with respect to $t$. Since $k^{\prime 2}+h^{\prime 2}=1$, we have $k^{\prime} k^{\prime \prime}+h^{\prime} h^{\prime \prime}=0$ and so

$$
K=-k^{\prime \prime} \operatorname{coth} k \text {. }
$$

Let us assume that $K$ is a positive constant, then

$$
\left(k^{\prime}\right)^{2}=C_{1}-2 K \ln \cosh k,
$$


where $C_{1}$ is a constant to be determined by the boundary conditions.

As $\alpha$ must cut the axis orthogonally, we can assume that the lowest point occurs at $t=0$. Then $k(0)=0$ and $k^{\prime}(0)=1$, thus $C_{1}=1$, that is

$$
\left(k^{\prime}\right)^{2}=1-2 K \ln \cosh k .
$$

Since the lowest point occurs at $t=0, h^{\prime}(t)>0$ and hence, by (5.2), $\lambda_{2}(t)>0$ for sufficiently small $t$. But $\lambda_{2}$ must have the same sign, so $h^{\prime}(t)>0$ for all $t$. Hence, $h$ increases as $t$ increases (and $S$ must be embedded).

Suppose $z$ is the highest point of $S$, then $k\left(t_{z}\right)=0$ for some $t_{z}>0$. Hence the domain of $t$ is $\left[0, t_{z}\right]$ and

$$
k(0)=k\left(t_{z}\right)=0, \quad h^{\prime}(0)=h^{\prime}\left(t_{z}\right)=0, \quad k^{\prime}(0)=1, \quad k^{\prime}\left(t_{z}\right)=-1 .
$$

On the other hand, by (5.3), $k^{\prime \prime}<0$ which implies that $k^{\prime}$ decreases from $k^{\prime}(0)=1$ to $k^{\prime}\left(t_{z}\right)=-1$. So, as $t$ increases from 0 to $t_{z}, k$ first increases from 0 to $k_{\max }=k\left(t_{\max }\right)$, for some $t_{\max } \in\left[0, t_{z}\right]$, then decreases from $k_{\max }$ to 0 .

Thus, $k$ must increase from 0 to $t_{\max }$ and $k^{\prime}\left(t_{\max }\right)=0$. So, from (5.4), one obtains $k\left(t_{\max }\right)=\cosh ^{-1} \exp (1 / 2 K)$.

Now, let $u=k^{\prime}$, where $-1 \leq u \leq 1$, then by equation (5.4) we have

$$
k=\cosh ^{-1} \exp \left(\frac{1-u^{2}}{2 K}\right), \quad-1 \leq u \leq 1 .
$$

Since $u=k^{\prime}, \frac{d u}{d t}=k^{\prime \prime}=-K \tanh k$, we have by (5.5)

$$
\frac{d h}{d u}=-\frac{1}{K} \frac{\sqrt{1-u^{2}}}{\sqrt{1-\exp \left(-\frac{1-u^{2}}{K}\right)}} .
$$

Then

$$
h=-\frac{1}{K} \int_{1}^{u} \frac{\sqrt{1-u^{2}}}{\sqrt{1-\exp \left(-\frac{1-u^{2}}{K}\right)}} d u+C, \quad-1 \leq u \leq 1,
$$

where $C$ is a real constant.

Also, we have

$$
\begin{aligned}
& h_{\min }=C, \\
& h_{\max }=\frac{1}{K} \int_{-1}^{1} \frac{\sqrt{1-u^{2}}}{\sqrt{1-\exp \left(-\frac{1-u^{2}}{K}\right)}} d u+C
\end{aligned}
$$


and

$$
h_{0}=\frac{1}{K} \int_{-1}^{0} \frac{\sqrt{1-u^{2}}}{\sqrt{1-\exp \left(-\frac{1-u^{2}}{K}\right)}} d u+C .
$$

Therefore, from (5.5) and (5.6), as $u$ decreases from 1 to $-1, h$ increases from $h_{\min }$ to $h_{\max }$, and $k$ first increases from 0 to $k_{\max }$ then decreases from $k_{\max }$ to 0 . Also, $S$ must be symmetric about $\mathbb{H}^{2} \times\left\{h_{0}\right\}$. Thus,

Proposition 5.1. Let $S$ be a complete immersed sphere of revolution (about the vertical line $\{1,0,0\} \times \mathbb{R})$ in $\mathbb{H}^{2} \times \mathbb{R}$ with constant $K>0$, given by

$$
\psi_{K}(u, v)=(\cosh k(u), \sinh k(u) \cos v, \sinh k(u) \sin v, h(u)),
$$

where $\alpha(u)=(\cosh k(u), \sinh k(u), 0, h(u))$ is the generating curve of $S$.

Then, $S$ must be embedded and the generating curve is given by

$$
\begin{aligned}
& k(u)=\cosh ^{-1} \exp \left(\frac{1-u^{2}}{2 K}\right), \\
& h(u)=-\frac{1}{K} \int_{1}^{u} \frac{\sqrt{1-u^{2}}}{\sqrt{1-\exp \left(-\frac{1-u^{2}}{K}\right)}} d u+C,
\end{aligned}
$$

where $-1 \leq u \leq 1$ and $C$ is a real constant (cf. Figure 11).

Also, $\mathbb{H}^{2} \times\left\{h_{0}\right\}$, where

$$
h_{0}=\frac{1}{K} \int_{-1}^{0} \frac{\sqrt{1-u^{2}}}{\sqrt{1-\exp \left(-\frac{1-u^{2}}{K}\right)}} d u+C,
$$

divides $S$ into two (upper and lower) symmetric parts.

Remark 5.1. Let us observe that the above analysis is the same, in spirit, as in [CR] for the case of revolution surfaces in $\mathbb{S}^{2} \times \mathbb{R}$ with constant positive extrinsic curvature. Moreover, we will need that result, so we will state it here:

Proposition 5.2. Let $S$ be a complete immersed sphere of revolution (about the vertical line $\{1,0,0\} \times \mathbb{R})$ in $\mathbb{S}^{2} \times \mathbb{R} \subset \mathbb{R}^{4}$ with constant $K>0$, given by

$$
\psi_{K}(u, v)=(\cos k(u), \sin k(u) \cos v, \sin k(u) \sin v, h(u)),
$$

where $\alpha(u)=(\cos k(u), \sin k(u), 0, h(u))$ is the generating curve of $S$. 


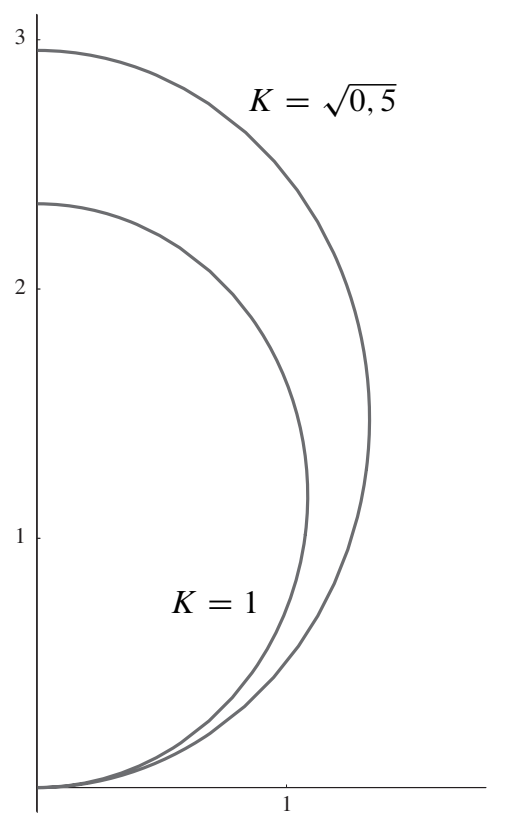

Figure 11. Profile curve of a rotational example.

Then,

i) $S$ must be embedded,

ii) $S$ stays in $\mathbb{D} \times \mathbb{R}$, where $\mathbb{D}$ denotes the open hemisphere of $\mathbb{S}^{2}$ of center $(1,0,0)$,

iii) the generating curve is given by

$$
\begin{aligned}
& k(u)=\cos ^{-1} \exp \left(-\frac{1-u^{2}}{2 K}\right), \\
& h(u)=-\frac{1}{K} \int_{1}^{u} \frac{\sqrt{1-u^{2}}}{\sqrt{\exp \left(\frac{1-u^{2}}{K}\right)-1}} d u+C,
\end{aligned}
$$

where $-1 \leq u \leq 1$ and $C$ is a real constant.

Also, $\mathbb{D} \times\left\{h_{0}\right\}$, where

$$
h_{0}=\frac{1}{K} \int_{-1}^{0} \frac{\sqrt{1-u^{2}}}{\sqrt{\exp \left(\frac{1-u^{2}}{K}\right)-1}} d u+C,
$$

divides $S$ into two (upper and lower) symmetric parts. 


\section{Vertical and horizontal height estimates for $K$-surfaces}

We divide this section in three parts. First, we establish some necessary equations for surfaces with positive extrinsic curvature in $\mathbb{M}^{2} \times \mathbb{R}, \mathbb{M}^{2}$ a Riemannian surface. Second, we obtain vertical height estimates for compact embedded surfaces with constant positive extrinsic curvature in $\mathbb{M}^{2} \times \mathbb{R}$ and boundary in a slice. And finally, we give horizontal height estimates for $K$-surfaces in $\mathbb{H}^{2} \times \mathbb{R}$ and boundary on a vertical plane.

6.1. Necessary equations. We will work in the spirit of [AEG2] but using the conformal structure induced by the second fundamental form of the surface as in [AEG3].

Let us denote by $g$ the metric of $\mathbb{M}^{2}$. Then the metric of $\mathbb{M}^{2} \times \mathbb{R}$ is given by $\langle\rangle=,g+d t^{2}$. Let $\psi: S \rightarrow \mathbb{M}^{2} \times \mathbb{R}$ be an immersion with positive extrinsic curvature $K$ with unit normal vector field $N$.

Let $\pi: \mathbb{M}^{2} \times \mathbb{R} \rightarrow \mathbb{M}^{2}$ and $\pi_{\mathbb{R}}: \mathbb{M}^{2} \times \mathbb{R} \rightarrow \mathbb{R}$ be the usual projections. We denote by $h: S \rightarrow \mathbb{R}$ the height function, that is, $h(z)=\pi_{\mathbb{R}}(\psi(z))$, and $v=\left\langle N, \frac{\partial}{\partial t}\right\rangle$, $\frac{\partial}{\partial t}$ the gradient in $\mathbb{M}^{2} \times \mathbb{R}$ of the function $t$.

Since $K>0$ the second fundamental form II is definite (and positive definite for a suitable normal $N$ ). Then, we can choose a conformal parameter $z$ such that the fundamental forms I and II can be written as

$$
\begin{gathered}
\mathrm{I}=\langle d \psi, d \psi\rangle=E d z^{2}+2 F|d z|^{2}+\bar{E} d \bar{z}^{2}, \\
\mathrm{II}=-\langle d \psi, d N\rangle=2 \rho|d z|^{2}, \quad \rho>0 .
\end{gathered}
$$

Here

$$
K=-\frac{\rho^{2}}{D}
$$

with $D=|E|^{2}-F^{2}<0$. The mean curvature of $S$ is

$$
H=-\frac{F \rho}{D}=\frac{K}{\rho} F
$$

Let us write

$$
\frac{\partial}{\partial t}=T+v N
$$

where $T$ is a tangent vector field on $S$. Since $\frac{\partial}{\partial t}$ is the gradient in $\mathbb{M}^{2} \times \mathbb{R}$ of the function $t$, it follows that $T$ is the gradient of $h$ on $S$. Thus, from (6.1), one gets

$$
T=\frac{1}{D}\left(\alpha \partial_{z}+\bar{\alpha} \partial_{\bar{z}}\right)
$$

where

$$
\alpha=\bar{E} h_{z}-F h_{\bar{z}}
$$


In addition, we obtain the following equations:

$$
\begin{aligned}
\langle T, T\rangle & =\frac{1}{D}\left(\alpha h_{z}+\bar{\alpha} h_{\bar{z}}\right), \\
\langle T, T\rangle & =\frac{1}{D}\left(E h_{\bar{z}}^{2}+\bar{E} h_{z}^{2}-2 F\left|h_{z}\right|^{2}\right), \\
h_{z} & =\frac{1}{D}(E \alpha+F \bar{\alpha}), \\
\left|h_{z}\right|^{2} & =\|T\|^{2} F+\frac{|\alpha|^{2}}{D} .
\end{aligned}
$$

Lemma 6.1. Let $\psi: S \rightarrow \mathbb{M}^{2} \times \mathbb{R}$ be an immersion with $K>0$. Then, for a conformal parameter $z$ for the second fundamental form, the following equations are satisfied:

$$
\begin{aligned}
& \frac{\rho_{\bar{z}}}{\rho}+\left(\Gamma_{12}^{1}-\Gamma_{22}^{2}\right)=\kappa \alpha \frac{v}{\rho} \quad(\text { Codazzi }), \\
& h_{z z}=\Gamma_{11}^{1} h_{z}+\Gamma_{11}^{2} h_{\bar{z}}, \\
& h_{z \bar{z}}=\Gamma_{12}^{1} h_{z}+\Gamma_{12}^{2} h_{\bar{z}}+v \rho, \\
& v_{\bar{z}}=\frac{\alpha K}{\rho} \\
& \|T\|^{2}+v^{2}=1 .
\end{aligned}
$$

Here $\kappa(p)$ stands for the Gauss curvature of $\mathbb{M}^{2}$ at $\pi(\psi(p))$, and $\Gamma_{i j}^{k}, i, j, k=$ 1,2 , are the Christoffel symbols associated to $z$.

Proof. From (6.1) we have

$$
\begin{aligned}
\nabla_{\partial_{z}} \partial_{z} & =\Gamma_{11}^{1} \partial_{z}+\Gamma_{11}^{2} \partial_{\bar{z}}, \\
\nabla_{\partial_{z}} \partial_{\bar{z}} & =\Gamma_{12}^{1} \partial_{z}+\Gamma_{12}^{2} \partial_{\bar{z}}+\rho N, \\
-\nabla_{\partial_{\bar{z}}} N & =\frac{K}{\rho}\left(\bar{E} \partial_{z}-F \partial_{\bar{z}}\right) .
\end{aligned}
$$

Thus, the scalar product of these equalities with $\frac{\partial}{\partial t}$ gives us $(6.11),(6.12)$ and (6.13), respectively.

The last equation follows from

$$
1=\left\langle\frac{\partial}{\partial t}, \frac{\partial}{\partial t}\right\rangle=\langle T, T\rangle+v^{2}
$$

Finally, from (6.15) we get

$$
\left\langle\nabla_{\partial_{\bar{z}}} \nabla_{\partial_{z}} \partial_{z}-\nabla_{\partial_{z}} \nabla_{\partial_{\bar{z}}} \partial_{z}, N\right\rangle=\rho_{\bar{z}}+\rho\left(\Gamma_{12}^{1}-\Gamma_{22}^{2}\right) .
$$


Hence, using the relationship between the curvature tensors of a product manifold (see, for instance, [O, p. 210]), the Codazzi equation becomes

$$
\kappa \alpha \frac{\nu}{\rho}=\frac{\rho_{\bar{z}}}{\rho}+\left(\Gamma_{12}^{1}-\Gamma_{22}^{2}\right) .
$$

That is, (6.10) holds.

Remark 6.1. The equation (6.14) will be used subsequently without comment.

From now on we will assume that $K$ is a positive constant on $S$. A straightforward computation gives us

$$
\Gamma_{12}^{1}+\Gamma_{22}^{2}=\frac{D_{\bar{z}}}{2 D}
$$

Thus, from (6.10),

$$
\frac{D_{\bar{z}}}{2 D}-\frac{\rho_{\bar{z}}}{\rho}=2 \Gamma_{12}^{1}-\kappa \alpha \frac{\nu}{\rho} .
$$

Since $K$ is constant, we obtain from (6.2) that

$$
\frac{D_{\bar{z}}}{2 D}-\frac{\rho_{\bar{z}}}{\rho}=0
$$

and

$$
\Gamma_{12}^{1}=\kappa \alpha \frac{v}{2 \rho} .
$$

Using $\Gamma_{12}^{2}=\overline{\Gamma_{12}^{1}},(6.18),(6.8)$ and (6.2), one has

$$
\begin{aligned}
E_{\bar{z}} & =2\left\langle\nabla_{\partial_{\bar{z}}} \partial_{z}, \partial_{z}\right\rangle=2\left(E \Gamma_{12}^{1}+F \Gamma_{12}^{2}\right) \\
& =\kappa \frac{\nu}{\rho}(E \alpha+F \bar{\alpha})=\kappa \frac{\nu D}{\rho} h_{z}=-\kappa \frac{\nu \rho}{K} h_{z} .
\end{aligned}
$$

On the other hand, by using (6.12), (6.18), (6.6) and (6.2),

$$
\begin{aligned}
2 h_{z \bar{z}} & =\kappa \frac{v}{\rho}\left(\alpha h_{z}+\bar{\alpha} h_{\bar{z}}\right)+2 v \rho=\kappa \frac{v}{\rho} D\|T\|^{2}+2 v \rho \\
& =-\kappa \frac{v \rho}{K}\left(1-v^{2}\right)+2 v \rho=\frac{v \rho}{K}\left(2 K-\kappa\left(1-v^{2}\right)\right) .
\end{aligned}
$$

Now, we compute $v_{z \bar{z}}$. From (6.13) and (6.17),

$$
v_{z \bar{z}}=\alpha_{z} \frac{K}{\rho}-\alpha K \frac{\rho_{z}}{\rho^{2}}=\alpha_{z} \frac{K}{\rho}-\frac{\alpha K}{\rho} \frac{D_{z}}{2 D} .
$$

Hence, we need to compute $\alpha_{z}$ :

$$
\alpha_{z}=2\left\langle\nabla_{\partial_{z}} \partial_{\bar{z}}, \partial_{\bar{z}}\right\rangle h_{z}+\bar{E} h_{z z}-\left\langle\nabla_{\partial_{z}} \partial_{z}, \partial_{\bar{z}}\right\rangle h_{\bar{z}}-\left\langle\partial_{z}, \nabla_{\partial_{z}} \partial_{\bar{z}}\right\rangle h_{\bar{z}}-F h_{z \bar{z}},
$$


where we have used (6.5).

If one considers (6.12) and (6.11), then

$$
\begin{aligned}
2\left\langle\nabla_{\partial_{z}} \partial_{\bar{z}}, \partial_{\bar{z}}\right\rangle h_{z} & =2 h_{z} F \Gamma_{12}^{1}+2 h_{z} \bar{E} \Gamma_{12}^{2}, \\
\bar{E} h_{z z} & =h_{z} \bar{E} \Gamma_{11}^{1}+h_{\bar{z}} \bar{E} \Gamma_{11}^{2}, \\
-\left\langle\nabla_{\partial_{z}} \partial_{z}, \partial_{\bar{z}}\right\rangle h_{\bar{z}} & =-h_{\bar{z}} F \Gamma_{11}^{1}-h_{\bar{z}} \bar{E} \Gamma_{11}^{2}, \\
-\left\langle\partial_{z}, \nabla_{\partial_{z}} \partial_{\bar{z}}\right\rangle h_{\bar{z}} & =-h_{\bar{z}} E \Gamma_{12}^{1}-h_{\bar{z}} F \Gamma_{12}^{2}, \\
-F h_{z \bar{z}} & =-h_{z} F \Gamma_{12}^{1}-h_{\bar{z}} F \Gamma_{12}^{2}-v \rho F .
\end{aligned}
$$

Therefore,

$$
\alpha_{z}=\alpha\left(\Gamma_{12}^{2}+\Gamma_{11}^{1}\right)+\alpha \Gamma_{12}^{2}-\bar{\alpha} \Gamma_{12}^{1}-v \rho F=\alpha \frac{D_{z}}{2 D}-v \rho F,
$$

where (6.16) is used and that $\bar{\alpha} \Gamma_{12}^{1}$ is a real function from (6.18).

Finally, using (6.21) and (6.3),

$$
v_{z \bar{z}}=-K F v=-\rho H \nu .
$$

Hence, we have obtained the Laplacian of $h, v$ and the derivative of the $(2,0)$-part of I with respect to II. That is

Lemma 6.2. Let $\psi: S \rightarrow \mathbb{M}^{2} \times \mathbb{R}$ be an immersion with constant positive extrinsic curvature $K$ on $S$, then

$$
\begin{aligned}
\Delta^{\mathrm{II}} h & =\left(2 K-\kappa\left(1-v^{2}\right)\right) \frac{v}{K}, \\
\Delta^{\mathrm{II}} v & =-2 H v, \\
E_{\bar{z}} & =-\kappa \frac{v \rho}{K} h_{z} .
\end{aligned}
$$

Now, we define a quadratic form which will play an important role in the following sections. Let $\varepsilon$ be a constant equal to 1 or -1 . Then, we consider the new quadratic form

$$
A=\mathrm{I}+g(v) d h^{2}
$$

where $g(v)$ is the only solution to the ODE

$$
\phi(v)=\frac{v^{2}-1}{2} g^{\prime}(v)
$$

such that it is well defined for $v= \pm 1$, where

$$
\phi=\frac{v}{K}\left(\left(2 K+\varepsilon\left(v^{2}-1\right)\right) g-\varepsilon\right)-\left(1-v^{2}\right) g^{\prime} .
$$


That is, $g(v)$ is the real analytic function given by

$$
g(v)=\frac{v^{2}-1+\varepsilon K\left(e^{\frac{\varepsilon\left(1-v^{2}\right)}{K}}-1\right)}{\left(1-v^{2}\right)^{2}} .
$$

Remark 6.2. In order to show the above assertion, observe that the function $\tilde{g}: \mathbb{R} \rightarrow \mathbb{R}$,

$$
\tilde{g}(t)=\frac{t^{2}-1+\varepsilon K\left(e^{\frac{\varepsilon\left(1-t^{2}\right)}{K}}-1\right)}{\left(1-t^{2}\right)^{2}},
$$

is well defined for each $t \in \mathbb{R}$ (in particular, if $t= \pm 1$ ) and it is an analytic function. This is easy to see bearing in mind that

$$
e^{t}=1+\sum_{n=1}^{\infty} \frac{t^{n}}{n !} .
$$

In addition

$$
\frac{\varepsilon}{2 K}=g( \pm 1) \leq g(v) \leq g(0)=-1+\varepsilon K\left(e^{\frac{\varepsilon}{K}}-1\right) .
$$

Let us observe that

$$
\chi(v)=1+g(v)\|T\|^{2}=\frac{\varepsilon K\left(e^{\frac{\varepsilon\left(1-v^{2}\right)}{K}}-1\right)}{1-v^{2}}
$$

satisfies

$$
\begin{array}{ll}
1=\chi( \pm 1) \leq \chi(v) & \text { if } \varepsilon=1, \\
0<K\left(1-e^{\frac{-1}{K}}\right)=\chi(0) \leq \chi(\nu) & \text { if } \varepsilon=-1
\end{array}
$$

for all $-1 \leq v \leq 1$.

Let us denote

$$
Q=E+g(v) h_{z}^{2},
$$

then $Q d z^{2}$ can be considered as the (2,0)-part of the real quadratic form $A$ for the second fundamental form II.

The extrinsic curvature of the pair (II, $A$ ) (see [Mi]) is given by

$$
\begin{aligned}
K(\mathrm{II}, A) & =\frac{\left(F+g\left|h_{z}\right|^{2}\right)^{2}-|Q|^{2}}{\rho^{2}} \\
& =\frac{\left(F^{2}-|E|^{2}\right)+g\left(2 F\left|h_{z}\right|^{2}-E h_{\bar{z}}^{2}-\bar{E} h_{z}^{2}\right)}{\rho^{2}} \\
& =\frac{1}{K}+\frac{g}{K}\|T\|^{2}=\frac{1}{K}\left(1+g\|T\|^{2}\right),
\end{aligned}
$$

where we have used (6.2) and (6.7).

In particular, the previous computation gives us

$$
|Q|^{2}=\left(F+g\left|h_{z}\right|^{2}\right)^{2}+D\left(1+g\|T\|^{2}\right) .
$$


6.2. Vertical height estimates in $\mathbb{M}^{2} \times \mathbb{R}$. Here, we establish upper bounds for compact graphs with positive constant extrinsic curvature and boundary in a slice of a product space $\mathbb{M}^{2} \times \mathbb{R}$.

Theorem 6.1. Let $\mathbb{M}^{2}$ be a Riemannian surface, $\psi: S \rightarrow \mathbb{M}^{2} \times \mathbb{R}$ be a compact graph on a domain $\Omega \subset \mathbb{M}^{2}$, with positive constant extrinsic curvature $K$ and whose boundary is contained in the slice $\mathbb{M}^{2} \times\{0\}$. Let $k$ be the minimum of the Gauss curvature on $\Omega \subset \mathbb{M}^{2}$. Then, there exists a constant $c_{K}$ (depending only on $K$ and $k$ ) such that $|h(p)| \leq c_{K}$ for all $p \in S$.

Proof. We want to compute the Laplacian of a certain function given by $h+f(v)$ for a suitable real function $f$. Since, we know $h_{z \bar{z}}$ from (6.20) then we focus our attention on $f(v)_{z \bar{z}}$. We can assume $h \geq 0$.

By using (6.13), (6.22), (6.2) and (6.9),

$$
\begin{aligned}
f(v)_{z \bar{z}} & =f^{\prime}(v) v_{z \bar{z}}+f^{\prime \prime}(v)\left|v_{z}\right|^{2}=-f^{\prime}(v) K F v+f^{\prime \prime}(v) \frac{|\alpha|^{2} K^{2}}{\rho^{2}} \\
& =-f^{\prime}(v) K F v-f^{\prime \prime}(v) K\left(\left|h_{z}\right|^{2}-\left(1-v^{2}\right) F\right) \\
& =-K\left(F\left(v f^{\prime}(v)-\left(1-v^{2}\right) f^{\prime \prime}(v)\right)+\left|h_{z}\right|^{2} f^{\prime \prime}(v)\right) .
\end{aligned}
$$

First, note that since II must be positive definite and $S$ is a graph, then $v \leq 0$. Second, we distinguish two cases, $k=0$ and $k \neq 0$. When $k=0$, we consider

$$
f(v)=\frac{v}{\sqrt{K}},
$$

thus

$$
f(v)_{z \bar{z}}=-\sqrt{K} F v
$$

and, from (6.2),

$$
\begin{aligned}
(h+f(v))_{z \bar{z}} & =\frac{2 K-\kappa\left(1-v^{2}\right)}{2 K} v \rho-\sqrt{K} F v \\
& =-\frac{\kappa}{2 K}\left(1-v^{2}\right) v \rho+(\sqrt{-D}-F) v \sqrt{K} \\
& \geq-\frac{\kappa}{2 K}\left(1-v^{2}\right) v \rho \geq 0
\end{aligned}
$$

thus, one has $\Delta^{\mathrm{II}}(h+f(v)) \geq 0$ on our surface and $h+f(v) \leq 0$ on the boundary, so $h \leq-v / \sqrt{K} \leq 1 / \sqrt{K}$.

When $k \neq 0$, we can suppose that $k=\varepsilon$, where $\varepsilon$ is -1 or 1 . To do that it is enough to consider, the new metric on $\mathbb{M}^{2} \times \mathbb{R}$ given by the quadratic form $|k| g+d t^{2}$ and the surface $S^{\prime}=\left\{(x, \sqrt{|k|} t) \in \mathbb{M}^{2} \times \mathbb{R}:(x, t) \in S\right\}$ which has constant extrinsic curvature $K /|k|$. Here, $g$ denotes the induced metric on $\mathbb{M}^{2}$. 
We consider

$$
f^{\prime}(v)=\sqrt{\varepsilon \frac{1-e^{-\varepsilon \frac{1-v^{2}}{K}}}{1-v^{2}}} .
$$

This function is real analytic and so is every primitive $f(v)$.

In addition

$$
f^{\prime \prime}(v)=\frac{v e^{-\varepsilon \frac{1-v^{2}}{K}}}{K} \frac{g(v)}{f^{\prime}(v)}
$$

where $g(v)$ is given by (6.24).

Thus,

$$
v f^{\prime}(v)-\left(1-v^{2}\right) f^{\prime \prime}(v)=\frac{v e^{-\varepsilon \frac{1-v^{2}}{K}}}{K f^{\prime}(v)}
$$

and

$$
f(v)_{z \bar{z}}=-\frac{v e^{-\varepsilon \frac{1-v^{2}}{K}}}{f^{\prime}(v)}\left(F+g(v)\left|h_{z}\right|^{2}\right) .
$$

We observe that $\left(F+g(v)\left|h_{z}\right|^{2}\right)|d z|^{2}$ is the $(1,1)$-part of the quadratic form $A$, given by (6.23), and our quadratic form $Q d z^{2}$, given by (6.27), is the (2,0)-part of $A$ for II.

Moreover, from (6.20) and (6.28),

$$
(h+f(v))_{z \bar{z}}=\frac{2 K-\kappa\left(1-v^{2}\right)}{2 K} v \rho-\frac{v e^{-\varepsilon \frac{1-v^{2}}{K}}}{f^{\prime}(v)} \sqrt{\frac{\rho^{2}\left(1+g(v)\left(1-v^{2}\right)\right)}{K}+|Q|^{2}}
$$

Here, we have used that $F+g(v)\left|h_{z}\right|^{2}>0$. This fact is clear because $K(\mathrm{II}, A)$ is positive from (6.28), (6.25) and (6.26), so, $A$ is positive definite or negative definite. Thus, $F+g(v)\left|h_{z}\right|^{2}$ is positive at every point or is negative everywhere. But, it is clear that it is positive at a highest point ( $h_{z}=0$ at this point).

Hence,

$$
\begin{aligned}
(h+f(v))_{z \bar{z}} & \geq \frac{2 K-\kappa\left(1-v^{2}\right)}{2 K} v \rho-\frac{v e^{-\varepsilon \frac{1-v^{2}}{K}}}{f^{\prime}(v)} \sqrt{\frac{\rho^{2}\left(1+g(v)\left(1-v^{2}\right)\right)}{K}} \\
& =\left(\frac{2 K-\kappa\left(1-v^{2}\right)}{2 K}-e^{-\varepsilon \frac{1-v^{2}}{2 K}}\right) v \rho \\
& \geq-\left(e^{-\varepsilon \frac{1-v^{2}}{2 K}}-1+\varepsilon \frac{1-v^{2}}{2 K}\right) v \rho \geq 0 .
\end{aligned}
$$

Here, we use that the term between parenthesis is non-negative. This is because the real function $e^{t}-1-t$ is non-negative everywhere. 
By taking,

$$
f(v)=\int_{0}^{v} f^{\prime}(t) d t
$$

one has $\Delta^{\mathrm{II}}(h+f(v)) \geq 0$ on the surface and $h+f(v) \leq 0$ on the boundary (because $f^{\prime}(v) \geq 0$ and $\left.v \leq 0\right)$.

Hence, the maximum height is less than or equal to

$$
c_{K}:=\int_{-1}^{0} f^{\prime}(t) d t
$$

Remark 6.3. It is clear that the height estimate $c_{K}$, when $k \neq 0$, is not reached for any graph with positive constant $K$ and boundary on a slice since

$$
e^{-\varepsilon \frac{1-v^{2}}{2 K}}-1+\varepsilon \frac{1-v^{2}}{2 K}
$$

is positive for $v \neq-1$ as well as $\Delta^{\mathrm{II}}(h+\phi(v))$. But then the maximum principle at the highest point shows that $\Delta^{\mathrm{II}}(h+\phi(v))$ vanishes identically; a contradiction.

But, when $k=0$, by (6.30), if the maximum height is attained at a point, then $h-v / \sqrt{K}$ vanishes identically on $S$. Thus, using (6.30) again, $\kappa$ and $E$ vanish identically. That is, the domain $\Omega$ is flat and $S$ is totally umbilical.

As a standard consequence of the Alexandrov reflection principle with respect to the slices $\mathbb{M}^{2} \times\left\{t_{0}\right\}$, we have the following corollary.

Corollary 6.1. Let $\psi: S \rightarrow \mathbb{M}^{2} \times \mathbb{R}$ be a compact embedded surface with positive constant extrinsic curvature $K$ and whose boundary is contained in the slice $\mathbb{M}^{2} \times\{0\}$. Let $k$ be the infimum of the Gauss curvature on $\mathbb{M}^{2}$. Then, there exists a constant $c_{K}$ (depending only on $K$ and $k$ ) such that $|h(p)| \leq 2 c_{K}$ for all $p \in S$.

We also observe that if $S$ is a non-compact properly embedded $K$-surface without boundary in $\mathbb{M}^{2} \times \mathbb{R}$ and $\mathbb{M}^{2}$ is compact then $S$ must have at least one top end and one bottom end. This is a consequence of our height estimates (see, for instance, [HLR]).

6.3. Horizontal height estimates. Now, we consider a compact embedded $K$-surface in $\mathbb{H}^{2} \times \mathbb{R}$ with boundary on a vertical plane and obtain distance estimates to this plane.

Theorem 6.2. Let $S$ be a compact embedded surface in $\mathbb{H}^{2} \times \mathbb{R}$, with extrinsic curvature a constant $K>0$. Let $P$ be a vertical plane in $\mathbb{H}^{2} \times \mathbb{R}$ and assume that 
$\partial S \subset P$. Then the distance from $S$ to $P$ is bounded; i.e., there is a constant $d$, independent of $S$, such that

$$
\operatorname{dist}(q, P) \leq d \quad \text { for all } q \in S .
$$

Proof. Let $q \in S$ be a furthest point from $P$. Up to isometry, we can assume $q \in \mathbb{H}^{2} \times\{0\}$ and $q \in \operatorname{ext}_{\mathbb{H}^{2} \times \mathbb{R}}(P)$. Let $P_{\gamma}(t)$ be the foliation of vertical planes along $\gamma$ with $P_{\gamma}(0)=P$ and $q \in P_{\gamma}(h)$. Let $X$ denote the horizontal Killing field of $\mathbb{H}^{2} \times \mathbb{R}$ generated by translations along $\gamma\left(X\right.$ is tangent to each $\mathbb{H}^{2} \times\{\tau\}$ and is translation along $\gamma \times\{\tau\}) ; X$ is orthogonal to the planes $P_{\gamma}(t)$.

Now, do Alexandrov reflection with the planes $P_{\gamma}(t)$, starting at $t=h$, and decrease $t$. For $h / 2<t \leq h$, the symmetry of the part $S$ in $\operatorname{ext}_{\mathbb{H}^{2} \times \mathbb{R}}\left(P_{\gamma}(t)\right)$ does not touch $\partial S$, since $\partial S \subset P$. Hence the Alexandrov reflection technique shows that the symmetry of $S^{+}(t)=S \cap \operatorname{ext}_{\mathbb{H}^{2} \times \mathbb{R}}\left(P_{\gamma}(t)\right)$, by $P_{\gamma}(t)$, intersects $S$ only at $S \cap P_{\gamma}(t)$ and $S$ is never orthogonal to $P_{\gamma}(s)$ for $t \leq s \leq h$. Since $X$ is orthogonal to each $P_{\gamma}(t)$, we conclude that $X$ is transverse to $S^{+}(h / 2)$, and $S^{+}(h / 2)$ is a graph over a domain of $P_{\gamma}(h / 2)$ with respect to the orbits of $X$.

Thus, to prove the theorem, it suffices to prove that $X$-graphs are a bounded distance from $P$, assuming the boundary of the graph is in $P$.

Now, suppose $S$ is an $X$-graph over a domain $D \subset P$ and chose $P_{\gamma}(t)$ as before. Let $S_{R}$ be the rotationally invariant sphere whose extrinsic curvature $K$ is the same as that of $S$. Denote by $c=c(K)$, the diameter of $S_{R}$.

We will now prove that for each $t>2 c$, the diameter of each connected component of $S(t)=P(t) \cap S$ is at most $2 c$. Suppose not, so for some component $C(t)$ of $S(t)$, there are points $x, y$ inside the domain $D(t)$ of $P(t)$ bounded by $C(t)$ with $\operatorname{dist}(x, y)>2 c$. Let $Q$ be the bounded domain of $\mathbb{H}^{2} \times \mathbb{R}$ bounded by $S \cup D$. Let $\beta$ be a path in $D(t)$ joining $x$ to $y, \beta$ is disjoint from $C(t)$. Let $\Omega$ be the "rectangle" formed by the orbits of $X$ joining $\beta$ to $P ; \Omega \subset Q$. Let $p$ be a point of $\Omega$ whose distance to $\partial \Omega$ is greater than $c ; p$ exists by construction of $\Omega$.

Let $\eta$ be the geodesic through $p$, each of whose points is a distance greater than $c$ from $\partial \Omega$; it is easy to find such an $\eta$ in the plane $P(t)$ containing $p$. The geodesic $\eta$ "enters" $Q$ at a first point $q_{0}$ and "leaves" $Q$ at a last point $q_{1}$.

Now, consider the family of spheres centered at each point of $\eta$, each sphere obtained from the rotational sphere $S_{R}$ (of extrinsic curvature $K$ ) by a translation of $\mathbb{H}^{2} \times \mathbb{R}$. Consider the family of spheres as entering $Q$ at $q_{0}$ (cf. Figure 12).

Then, there is some first sphere in the family (coming from $q_{0}$ ) that touches $\Omega$ for the first moment at an interior point of $\Omega$. Then the sphere passes through $\Omega$, not touching $\partial Q$ initially, and the sphere passes through $\Omega$ without touching $\partial \Omega$. Since the spheres leave $Q$ at $q_{1}$, there is some sphere that touches $\partial Q \cap S$ at a first point of contact. At this first point of contact (cf. Figure 13), the mean curvature vectors of $S$ and the rotational sphere are equal. Hence $S$ equals this sphere by the maximum principle; a contradiction. 

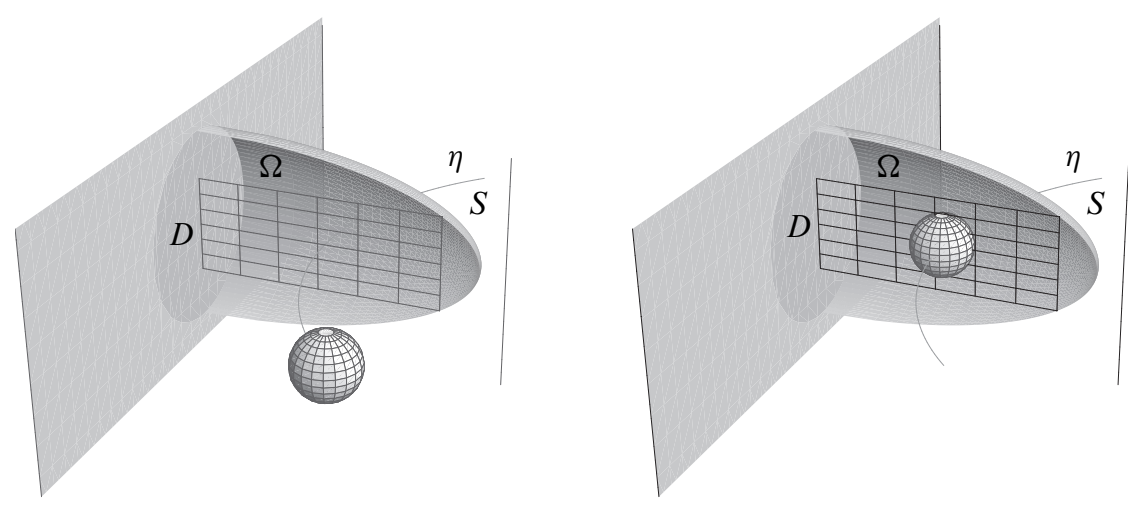

Figure 12. Moving the spheres along $\eta$.
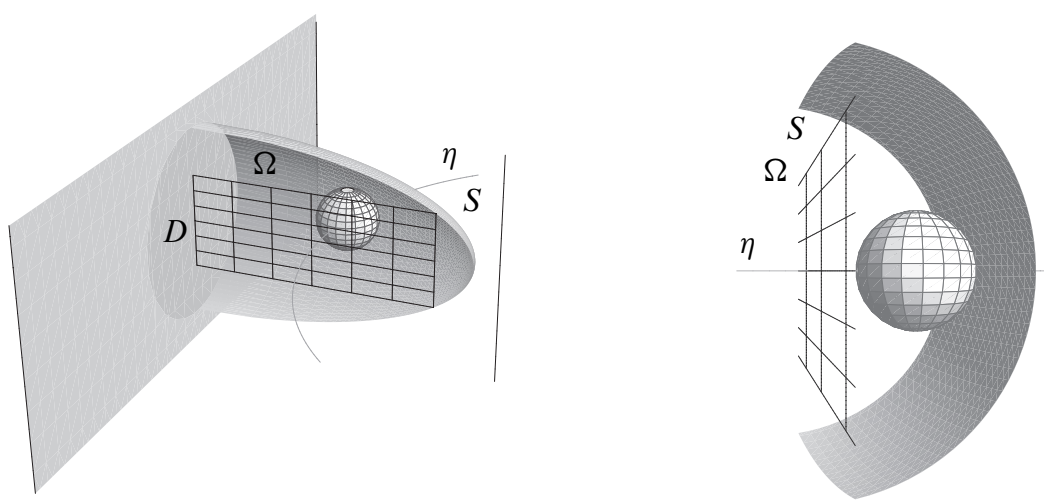

Figure 13. Touching $\partial Q$.

Now, if the theorem is false, there is a sequence of graphs $S_{n}$ over domains $D_{n} \subset P$, with diameter $\left(D_{n}\right)<2 c$ and $\operatorname{dist}\left(S_{n}, P\right)$ unbounded.

After an ambient isometry we can assume that the $D_{n}$ are contained in a fixed disk $D$ and the $S_{n}$ are contained in the horizontal Killing cylinder $C$ over $D$, i.e., a tubular neighborhood of a horizontal geodesic $\gamma$. We will use "tilted" vertical planes to show this is impossible.

We can assume, without lost of generality, that $\gamma=\{0, \pi\}, P$ is the vertical plane over the geodesic $\{\pi / 2,3 \pi / 2\}$, and the graphs $S_{n}$ satisfy $\pi\left(S_{n}\right)$ are asymptotic to 0 . 
Consider the vertical plane $Q\left(s_{1}, s_{2}\right)=\left\{s_{1}, s_{2}\right\} \times \mathbb{R}$. A simple calculation shows that for $s_{1}=0$ and $s_{2}$ positive and close to 0 , the symmetry through $Q\left(0, s_{2}\right)$ of $\zeta \cap \operatorname{int}_{\mathbb{H}^{2} \times \mathbb{R}}\left(Q\left(0, s_{2}\right)\right)$ does not intersect $P$. In particular, the symmetry of the part of any $S_{n} \cap \operatorname{int}_{\mathbb{H}^{2} \times \mathbb{R}}\left(Q\left(0, s_{2}\right)\right)$ does not intersect $\partial S_{n} \subset D$. By continuity, for $s_{1}$ negative and sufficiently close to 0 , the above last two statements continue to hold, i.e., the symmetry through $Q\left(s_{1}, s_{2}\right)$ of $\mathcal{C} \cap \operatorname{int}_{\mathbb{H}^{2} \times \mathbb{R}}\left(Q\left(s_{1}, s_{2}\right)\right)$ does not intersect $P$, and the symmetry of the part of any $S_{n} \cap \operatorname{int}_{\mathbb{H}^{2} \times \mathbb{R}}\left(Q\left(s_{1}, s_{2}\right)\right)$ does not intersect $\partial S_{n} \subset D$.

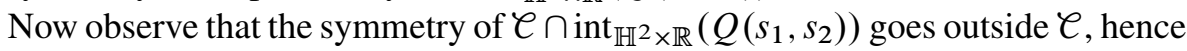

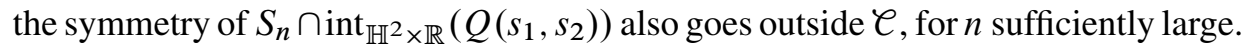

Choose $a$ and $b$ between $s_{1}$ and $s_{2}$ so that $Q(a, b)$ is disjoint from $C$. Let $R(t)$ be a foliation by vertical planes of the region of $\mathbb{H}^{2} \times \mathbb{R}$ between $Q(a, b)$ and $Q\left(s_{1}, s_{2}\right)$ with $R(0)=Q(a, b)$ and $R(1)=Q\left(s_{1}, s_{2}\right), 0 \leq t \leq 1$.

Consider doing Alexandrov reflection with the planes $R(t)$. Choose $n$ large so that the symmetry of $\left(S_{n}\right) \cap \operatorname{int}_{\mathbb{H}^{2} \times \mathbb{R}}\left(Q\left(s_{1}, s_{2}\right)\right)$ has points outside $\mathcal{C}$. The symmetry of this part of $S(n)$ through each $R(t)$ is disjoint from $P$, hence disjoint from $\partial S_{n}$. Also the symmetry of this part of $S(n)$ through $R(1)$ goes outside $\mathcal{C}$, and $R(0)$ is disjoint from $\mathcal{C}$ (cf. Figures 14 and 15).
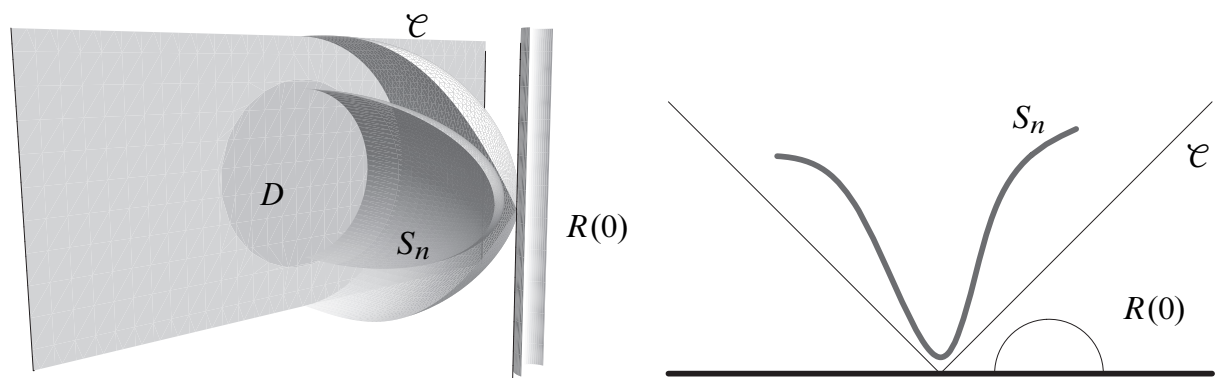

Figure 14. No accident of Alexandrov Reflection. The picture on the right is a horizontal section.

Hence there is a smallest $t$ such that the symmetry of $S(n)$ through $R(t)$ touches $S(n)$ at some point. Thus $R(t)$ is a symmetry plane of $S(n)$, which is a contradiction. This completes the proof.

This proof also works for properly embedded surfaces with constant mean curvature greater than $1 / 2$. Thus, [NR, Theorem 1.2] can be extended for $H$-surfaces with $H>1 / 2$, that is,

Corollary 6.2. Let $H>1 / 2$ and let $S$ be a properly embedded $H$-surface in $\mathbb{H}^{2} \times \mathbb{R}$ with finite topology and one end. Then $S$ is contained in a vertical cylinder of $\mathbb{H}^{2} \times \mathbb{R}$.

In Theorem 7.2, we use this corollary to prove that no such surface exists. 


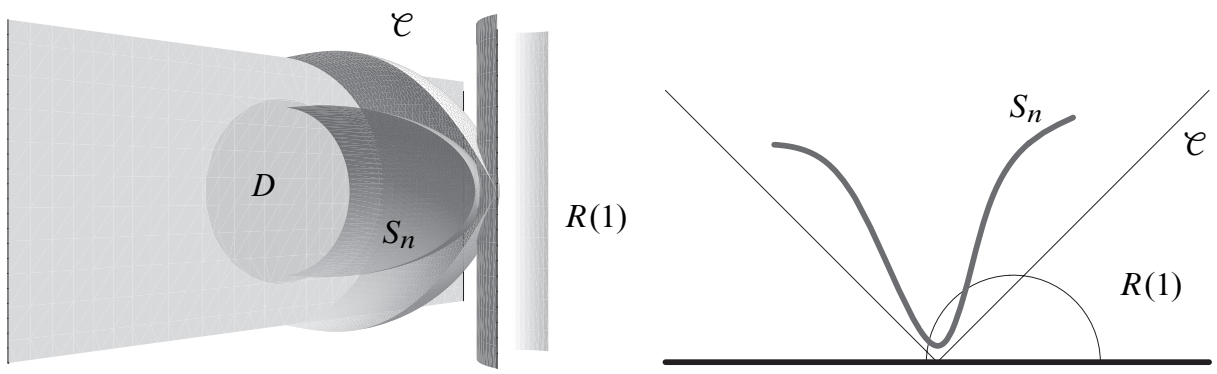

Figure 15. For $n$ large, the symmetry of $S_{n}$ goes outside $\ell$. The picture on the right is a horizontal section.

\section{Classification of complete $K$-surfaces in $\mathbb{H}^{2} \times \mathbb{R}$ and $\mathbb{S}^{2} \times \mathbb{R}$}

The aim of this section is to classify the complete surfaces with positive constant extrinsic curvature as the complete revolution examples established in Section 5.

In this section $\mathbb{M}^{2}(\varepsilon)$ stands for $\mathbb{S}^{2}$ or $\mathbb{H}^{2}$, depending on $\varepsilon=1$ or $\varepsilon=-1$, respectively. We continue working with a conformal parameter $z$ for the second fundamental form II. We now come to a key lemma.

Lemma 7.1. Let $\psi: S \rightarrow \mathbb{M}^{2}(\varepsilon) \times \mathbb{R}$ be an immersed $K$-surface. If we consider $S$ as the Riemann surface with the conformal structure induced by its second fundamental form, then the quadratic form given by (6.27) verifies

$$
\left|Q_{\bar{z}}\right|^{2} \leq \frac{K g^{\prime}(v)^{2}\left(1-v^{2}\right)^{2}\left|h_{z}\right|^{2}}{4 \chi(v)}|Q|^{2}
$$

where $g$ and $\chi$ are given by (6.24) and (6.25) respectively.

Proof. First, we compute the derivative of $Q$, which is given by

$$
Q_{\bar{z}}=E_{\bar{z}}+g^{\prime} \nu_{\bar{z}} h_{z}^{2}+2 g h_{z} h_{z \bar{z}} .
$$

So, from (6.19) and (6.20), one gets

$$
E_{\bar{z}}+2 g h_{z} h_{z \bar{z}}=\frac{v}{K}\left(\left(2 K+\varepsilon\left(v^{2}-1\right)\right) g-\varepsilon\right) \rho h_{z} .
$$

Moreover, (6.13), (6.8) and (6.6) give

$$
\begin{gathered}
\nu_{\bar{z}} E=-\rho \frac{E \alpha}{D}=\rho \frac{\bar{\alpha}}{D} F-\rho h_{z}, \\
\nu_{\bar{z}} h_{z}^{2}=-\rho h_{z} \frac{\alpha h_{z}}{D}=\rho \frac{\bar{\alpha}}{D}\left|h_{z}\right|^{2}-\|T\|^{2} \rho h_{z},
\end{gathered}
$$




$$
g^{\prime} \nu_{\bar{z}} h_{z}^{2}=g^{\prime}\left|h_{z}\right|^{2} \rho \frac{\bar{\alpha}}{D}-g^{\prime}\|T\|^{2} \rho h_{z} .
$$

Consequently,

$$
Q_{\bar{z}}=\rho g^{\prime}(v)\left(\frac{v^{2}-1}{2} h_{z}+\left|h_{z}\right|^{2} \frac{\bar{\alpha}}{D}\right) .
$$

So,

$$
\begin{aligned}
\left|Q_{\bar{z}}\right|^{2}= & \rho^{2} g^{\prime}(v)^{2}\left(\left(1-v^{2}\right)^{2} \frac{\left|h_{z}\right|^{2}}{4}\right. \\
& \left.-\left(1-v^{2}\right) \frac{\left|h_{z}\right|^{2}}{2}\left(\frac{\alpha h_{z}+\bar{\alpha} h_{\bar{z}}}{D}\right)+\left|h_{z}\right|^{4} \frac{|\alpha|^{2}}{D^{2}}\right) \\
= & \rho^{2} g^{\prime}(v)^{2}\left|h_{z}\right|^{2}\left(-\frac{\left(1-v^{2}\right)^{2}}{4}+\left|h_{z}\right|^{2} \frac{|\alpha|^{2}}{D^{2}}\right) \\
= & \frac{\rho^{2} g^{\prime}(v)^{2}\left|h_{z}\right|^{2}}{-D}\left(D \frac{\left(1-v^{2}\right)^{2}}{4}+\left|h_{z}\right|^{2} \frac{|\alpha|^{2}}{-D}\right) \\
= & K g^{\prime}(v)^{2}\left|h_{z}\right|^{2}\left(D \frac{\left(1-v^{2}\right)^{2}}{4}+\left|h_{z}\right|^{2}\left(\left(1-v^{2}\right) F-\left|h_{z}\right|^{2}\right)\right),
\end{aligned}
$$

where we have used (6.6), (6.2) and (6.9).

Thus, from (6.29)

$$
D=\frac{|Q|^{2}-\left(F+g(v)\left|h_{z}\right|^{2}\right)^{2}}{\chi(v)} .
$$

Hence, from the previous equation of $\left|Q_{\bar{z}}\right|^{2}$,

$$
\begin{aligned}
\left|Q_{\bar{z}}\right|^{2}= & K g^{\prime}(v)^{2}\left|h_{z}\right|^{2}\left(\frac{|Q|^{2}-\left(F+g(v)\left|h_{z}\right|^{2}\right)^{2}}{\chi(v)} \frac{\left(1-v^{2}\right)^{2}}{4}\right. \\
\left.+\left|h_{z}\right|^{2}\left(\left(1-v^{2}\right) F-\left|h_{z}\right|^{2}\right)\right) & \\
= & \frac{K g^{\prime}(v)^{2}\left(1-v^{2}\right)^{2}\left|h_{z}\right|^{2}}{4 \chi(v)}|Q|^{2} \\
& -\frac{K g^{\prime}(v)^{2}\left|h_{z}\right|^{2}}{4 \chi(v)}\left(\left(1-v^{2}\right)^{2}\left(F+g(v)\left|h_{z}\right|^{2}\right)^{2}\right. \\
& \left.-4 \chi(v)\left|h_{z}\right|^{2}\left(\left(1-v^{2}\right) F-\left|h_{z}\right|^{2}\right)\right) .
\end{aligned}
$$


Now, we show that the last term between parenthesis is non negative. That is,

$$
\begin{aligned}
\left(\left(1-v^{2}\right) F-\left(2+g(v)\left(1-v^{2}\right)\right)\left|h_{z}\right|^{2}\right)^{2} & \left(1-v^{2}\right)^{2} F^{2}-2 F\left|h_{z}\right|^{2}\left(2\left(1-v^{2}\right)+g(v)\left(1-v^{2}\right)^{2}\right) \\
= & \quad\left(4+4 g(v)\left(1-v^{2}\right)+g(v)^{2}\left(1-v^{2}\right)^{2}\right)\left|h_{z}\right|^{4} \\
= & \left(1-v^{2}\right)^{2}\left(F^{2}-2 F g(v)\left|h_{z}\right|^{2}+g(v)^{2}\left|h_{z}\right|^{4}\right) \\
& \quad+\left(1-v^{2}\right)\left|h_{z}\right|^{2}\left(-4 F+4 g(v)\left|h_{z}\right|^{2}\right)+4\left|h_{z}\right|^{4} \\
= & \left(1-v^{2}\right)^{2}\left(F+g(v)\left|h_{z}\right|^{2}\right)^{2}+4\left|h_{z}\right|^{2}\left(-F g(v)\left(1-v^{2}\right)^{2}\right. \\
& \left.\quad-F\left(1-v^{2}\right)+g(v)\left(1-v^{2}\right)\left|h_{z}\right|^{2}+\left|h_{z}\right|^{2}\right) \\
= & \left(1-v^{2}\right)^{2}\left(F+g(v)\left|h_{z}\right|^{2}\right)^{2}-4 \chi(v)\left|h_{z}\right|^{2}\left(\left(1-v^{2}\right) F-\left|h_{z}\right|^{2}\right)
\end{aligned}
$$

as we wanted to prove.

Therefore,

$$
\left|Q_{\bar{z}}\right|^{2} \leq \frac{K g^{\prime}(v)^{2}\left(1-v^{2}\right)^{2}\left|h_{z}\right|^{2}}{4 \chi(v)}|Q|^{2}
$$

This lemma shows that [J, Lemma 2.7.1, p. 75] can be used.

Lemma 7.2. Let $\psi: S \rightarrow \mathbb{M}^{2}(\varepsilon) \times \mathbb{R}$ be an immersion with positive constant extrinsic curvature. Then, the zeroes of $Q$ are isolated with negative index or $Q$ vanishes identically.

As a consequence, using the Poincaré Index Theorem, one has

Theorem 7.1. Let $\psi: S \rightarrow \mathbb{M}^{2}(\varepsilon) \times \mathbb{R}$ be an immersion with positive constant extrinsic curvature, with $S$ a topological sphere. Then $Q$ vanishes identically on $S$.

From Theorem 3.1 we know that every complete $K$-surface in $\mathbb{H}^{2} \times \mathbb{R}$ is properly embedded and it is compact or homeomorphic to $\mathbb{R}^{2}$. So, we will show that it cannot be homeomorphic to $\mathbb{R}^{2}$. This follows from the following result.

Theorem 7.2. For $K>0$ (or $H>1 / 2$ ) there is no properly embedded $K$-surface ( $H$-surface) in $\mathbb{H}^{2} \times \mathbb{R}$ with finite topology and one end.

Proof. We only outline the main steps of the proof since, in essence, it is the same as in [NR] for surfaces of constant mean curvature greater than $1 / \sqrt{3}$.

Note that the Plane Separation Lemma is valid for properly embedded surfaces with constant positive extrinsic curvature, so, Theorem 6.2 ensures us that such a 
surface must be contained in a vertical cylinder. Thus the Alexandrov reflection method using horizontal planes and Theorem 6.1 completes the proof as in [NR, Theorem 1.1].

Analogously, the result is also valid for $H$-surfaces, $H>1 / 2$, using height estimates in [AEG2] and Corollary 6.2.

Bringing this all together yields:

Theorem 7.3. A complete immersion with positive constant extrinsic curvature $K$ in $\mathbb{M}^{2}(\varepsilon) \times \mathbb{R}$ is a rotational sphere (cf. Section 5$)$.

Proof. By the Gauss equation [D], the Gauss curvature $K(\mathrm{I})$ of the surface satisfies

$$
K(\mathrm{I})=K+\varepsilon v^{2} \text {. }
$$

Thus, for $\varepsilon=1, K(\mathrm{I}) \geq K>0$ and, so, every complete $K$-surface in $\mathbb{S}^{2} \times \mathbb{R}$ must be a topological sphere from the Bonnet and Gauss-Bonnet theorems. On the other hand, from Theorem 3.1 and Theorem 7.2, we can also state that every complete $K$-surface in $\mathbb{H}^{2} \times \mathbb{R}$ must be a topological sphere.

Thus, by Theorem 7.1, $Q=0$ on any complete immersion with positive constant extrinsic curvature $K$ in $\mathbb{M}^{2}(\varepsilon) \times \mathbb{R}$.

Now we show that the immersion is rotational. Let us take doubly orthogonal coordinates $(u, v)$ for $(\mathrm{I}, \mathrm{II})$, that is,

$$
\begin{aligned}
\mathrm{I} & =m d u^{2}+n d v^{2}, \\
\mathrm{II} & =k_{1} m d u^{2}+k_{2} n d v^{2} .
\end{aligned}
$$

Then, since the metric II and the real quadratic form $A$ given by (6.23) are conformal (because $Q$ is the $(2,0)$-part of $A$ for II), we have that $h_{u} h_{v} \equiv 0$.

Thus, we can assume that $h_{u}$ vanishes locally. Using the compatibility equations given in [D] and making a suitable change of doubly orthogonal parameters, as in [AEG3, Theorem 3.1], one sees that all $m, n, k_{1}, k_{2}, h, v$ only depend on the second parameter $v$. Therefore, the uniqueness part in [D] gives that the immersion is invariant under a 1-parameter group of transformations $((u, v) \mapsto(u+t, v))$. To finish, as the surface is compact, then it must be invariant by the group of rotations, so Proposition 5.1 and Proposition 5.2 give us the result.

Note that Theorem 3.1 together with Theorem 7.2 shows that a complete $K$-surface $S$ in $\mathbb{H}^{2} \times \mathbb{R}$ must be embedded and topologically a sphere. Then, the Alexandrov reflection principle with respect to vertical planes proves $S$ is a rotational sphere. This gives us an alternative proof to Theorem 7.3 in $\mathbb{H}^{2} \times \mathbb{R}$.

Observe that a similar reasoning does not seem possible in $\mathbb{S}^{2} \times \mathbb{R}$. That is, the existence of the quadratic form $Q$ with isolated zeroes of negative index appears to be essential in this case. 
Concluding remarks. It would be interesting to know which of the results in this article extend to the other complete, simply connected, homogeneous 3-manifolds. For example, does the Hadamard-Stoker Theorem hold in Heisenberg space, the Berger spheres or the universal cover of $\operatorname{PSL}(2, \mathbb{R})$ ? The space $\mathrm{Sol}_{3}$ is foliated by totally geodesic surfaces, each isometric to $\mathbb{H}^{2}$ (in fact, there are two such orthogonal foliations, and their intersection is an Anosov flow). Using the above techniques, it is not hard to see that immersed compact surfaces of positive extrinsic curvature in $\mathrm{Sol}_{3}$ are embedded spheres. Also each leaf of the two orthogonal foliations is a symmetry submanifold by an ambient isometry of $\mathrm{Sol}_{3}$. Thus the Alexandrov reflection technique can be used to show that a compact embedded $H$-surface in $\mathrm{Sol}_{3}$ is a topological sphere. Alexandrov reflection with respect to one of the foliations shows it is a bigraph with respect to one of the leaves. Then using the orthogonal foliation it is also a bigraph with respect to an orthogonal leaf. Thus it is of genus zero; hence a sphere. Is this the case in Heisenberg space? If the extrinsic curvature $K$ is a positive constant, is the surface a rotational sphere?

\section{References}

[AR1] U. Abresch and H. Rosenberg, A Hopf Differential for Constant Mean Curvature Surfaces in $\mathbb{S}^{2} \times \mathbb{R}$ and $\mathbb{H}^{2} \times \mathbb{R}$. Acta Math. 193 (2004), 141-174. Zbl 1078.53053 MR 2134864

[AR2] U. Abresch and H. Rosenberg, Generalized Hopf differentials. Mat. Contemp. 28 (2005), 1-28. Zbl 1118.53036 MR 2195187

[AEG1] J. A. Aledo, J. M. Espinar and J. A. Gálvez, Complete surfaces of constant curvature in $\mathbb{H}^{2} \times \mathbb{R}$ and $\mathbb{S}^{2} \times \mathbb{R}$. Calc. Variations \& PDEs 29 (2007), 347-363. Zbl 1119.53039 MR 2321892

[AEG2] J. A. Aledo, J. M. Espinar and J. A. Gálvez, Height estimates for surfaces with positive constant mean curvature in $\mathbb{M}^{2} \times \mathbb{R}$. Illinois J. Math., to appear.

[AEG3] J. A. Aledo, J. M. Espinar and J. A. Gálvez, Surfaces with Constant Curvature in $\mathbb{S}^{2} \times \mathbb{R}$ and $\mathbb{H}^{2} \times \mathbb{R}$. Height Estimates and Representation. Bull. Braz. Math. Soc. (N.S.) 38 (2007), 533-554. Zbl 1136.53046 MR 2371944

[CW] M. P. do Carmo and F. W. Warner, Rigidity and convexity of hypersurfaces in spheres. J. Differential Geom. 4 (1970), 133-144. Zbl 0201.23702 MR 0266105

[CR] X. Cheng and H. Rosenberg, Embedded positive constant $r$-mean curvature hypersurfaces in $\mathbb{M}^{m} \times \mathbb{R}$. An. Acad. Brasil. Cienc. 72 (2005), 183-199. Zbl 1074.53049 MR 2137392

[C] R. J. Currier, On Hypersurfaces of Hyperbolic Space Infinitesimally Supported by Horospheres. Trans. Amer. Math. Soc. 313 (1989), 419-431. Zbl 0679.53045 MR 0935532

[D] B. Daniel, Isometric immersions into 3-dimensional homogeneous manifolds. Comment. Math. Helv., 82 (2007), 87-131. Zbl 1123.53029 MR 2296059 
[H] J. Hadamard, Sur certaines proprietes des trajectoires en dynamique. J. Math. Pures Appl. 3 (1897), 331-387. JFM 28.0643.01

[HLR] D. Hoffman, J. H. S. de Lira and H. Rosenberg, Constant mean curvature surfaces in $\mathbb{M}^{2} \times \mathbb{R}$. Trans. Amer. Math. Soc. 358 (2006), 491-507. Zbl 1079.53088 MR 2177028

[J] J. Jost, Two-dimensonal Geometric Variational problems. Pure Appl. Math. (New York), John Wiley \& Sons, Ltd., Chichester 1991.Zbl 0729.49001 MR 1100926

[Mi] T. K. Milnor, Abstract Weingarten Surfaces. J. Differential Geom. 15 (1980), 365-380. Zbl 0464.53002 MR 620893

[NR] B. Nelli and H. Rosenberg, Simply connected constant mean curvature surfaces in $\mathbb{H}^{2} \times \mathbb{R}$. Michigan Math. J. 54 (2006), 537-543. Zbl 1152.53307 MR 2280494

[O] B. O’Neill, Semi-Riemannian Geometry. Pure and Applied Math. 103, Academic Press, New York 1983. Zbl 0531.53051 MR 0719023

[S] J. Stoker, Über die Gestalt der positiv gekrümmten offenen Flächen im dreidimensionalen Raume. Compositio Math. 3 (1936), 55-88. Zbl 0013.27805 MR 1556933

Received May 23, 2007

José M. Espinar, Departamento de Geometría y Topología, Universidad de Granada, 18071 Granada, Spain

E-mail: jespinar@ugr.es

José A. Gálvez, Departamento de Geometría y Topología, Universidad de Granada, 18071 Granada, Spain

E-mail: jagalvez@ugr.es

Harold Rosenberg, Institut de Mathématiques, Université Paris VII, 2 place Jussieu, 75005 Paris, France

E-mail: rosen@math.jussieu 